\title{
Electrochemical growth of CoNi and Pt-CoNi soft magnetic composites on an alkanethiol monolayer-modified ITO substrate
}

D. Escalera-López, E. Gómez, E. Vallés*

Grup d'Electrodeposició de Capes Primes i Nanoestructures (Ge־CPN), Departament de Química Física and Institut de Nanociència i Nanotecnologia (IN $\left.{ }^{2} U B\right)$, Universitat de Barcelona, Martí i Franquès 1, 08028 Barcelona.

*Corresponding author

e.valles@ub.edu

Abstract

CoNi and Pt-CoNi magnetic layers on indium-tin oxide (ITO) substrates modified by an alkanethiol self-assembled monolayer (SAM) have been electrochemically obtained as an initial stage to prepare Semiconducting layer-SAM-Magnetic layer hybrid structures. The best conditions to obtain the maximum compactness of adsorbed layers of dodecanethiol (C12-SH) on ITO substrate have been studied using contact angle, AFM, XPS and electrochemical tests. The electrochemical characterization (electrochemical probe or voltammetric response in blank solutions) is fundamental to assure the maximum blocking of the substrate. Although the electrodeposition process on the SAMmodified ITO substrate is very slow if the blocking of the surface is significant, non-cracked metallic layers of $\mathrm{CoNi}$, with or without a previously electrodeposited seed-layer of platinum, have been obtained by optimizing the deposition potentials. Initial nucleation is expected to take place at the pinhole defects of the C12-SH SAM, followed by a mushroom-like growth regime through the SAM interface that allows the formation of a continuous metallic layer electrically connected to the ITO surface. Due to the potentiality of the methodology, the preparation of patterned metallic deposits on ITO substrate using SAMs with different coverage as templates is feasible.

Keywords: hybrid structures, electrodeposition, ITO-SAM substrate, magnetic layer, CoNi

1. Introduction 
The functionalisation and nanopatterning of substrates by means of self-assembled monolayers (SAMs) has been exploited for low-cost, high aspect ratio hierarchical growth applications in molecular spintronics ${ }^{1}$, selective adsorption of nanostructures ${ }^{2-6}$, biosensing ${ }^{7}$ and nanolithography ${ }^{8}$. Alkanethiol SAMs have been widely utilized for modifying conducting substrates, in particular welldefined Au surfaces ${ }^{9-17}$. Among all plausible nanomodifications, the formation of continuous metallic layers over SAM-modified Au electrodes has drawn a burgeoning interest. Several methodologies for obtaining a continuous metallic layer on the top of a SAM have been explored, the most performed being electrochemical methods. Unlike the cost-intensive physical methods such as vacuum layer deposition $^{18,19}$ or metal nano-transfer printing ${ }^{20-24}$, electrochemical methods stand out as an excellent choice due to their low cost and environment-friendly nature that allow easy tailoring of the composition, crystalline structure and thickness of metallic layers by an exhaustive control of both deposition conditions and electrolytic bath composition ${ }^{25}$. Kolb and co-workers intensively pursued the selective metallization of SAM surfaces by means of $\mathrm{Cu}$ or Ag underpotential deposition (UPD) ${ }^{26-29}$, but diffusion and nucleation of the metallic ions at the Au-SAM interface impeded the selective formation of a continuous film on the top of the SAM. The presence of nanopores, even in denselypacked alkanethiol SAMs, favoured the electrocrystallisation of the metal at the SAM pinholes due to inferior electrical resistance by means of a mushroom-type growth mechanism ${ }^{30,31}$. This phenomenon, undesired for obtaining metal-spacer-metal thin film hybrid structures, was used by Schilardi and coworkers to obtain thin metal films connected to the substrate by means of narrow wires obtained during the columnar growth ${ }^{32}$. Further studies revealed the possibility of using the SAM defects as a low-adherent support to nanopattern electrodeposited metallic films following the morphology of a templated substrate ${ }^{33,34}$.

Despite of the aforementioned, alkanethiol SAM formation on non-metallic surfaces has been scarcely studied, the most known examples being over $\mathrm{SnO}_{2}(110)^{35}$, manganite $\left(\mathrm{La}_{2 / 3} \mathrm{Sr}_{1 / 3} \mathrm{MnO}_{3}\right)^{36}$, magnetite $\left(\mathrm{Fe}_{3} \mathrm{O}_{4}\right)^{37}$ or fluorine-doped tin oxide ${ }^{38}$. One of the most promising substrates for such purpose is indium-tin oxide (ITO). ITO, a transparent semiconducting oxide, has extensively been used in numerous applications such as liquid-crystal displays (LCD), organic light-emitting diodes (OLEDs) ${ }^{39}$ and solar cells ${ }^{40}$ due to the combination of its optical transparency along with its electrical conductivity ${ }^{41}$. As a consequence, the formation of a highly-compact alkanethiol SAM above an ITO surface could easily modulate its electronic properties, thus expanding its application range. Several works have attempted to pursue this, with variable results: Grunze and co-workers studied the formation of octadecanethiol (C18-SH) or hexadecanethiol (C16-SH) SAMs over freshly-deposited or UV/ozone cleaned ITO substrates, by immersion in the neat liquid or in an ethanol solution, obtaining high-compactness $\mathrm{SAMs}^{42}$. Karsi et al. managed to obtain compact decanethiol (C10-SH), 
dodecanethiol (C12-SH) and octadecanethiol (C18-SH) SAMs over ITO commercial substrates by immersion in the neat liquid, using a pre-treatment method alternative to UV/ozone ${ }^{43}$. However, the electrochemical characterization of the substrates to evaluate defects in the SAM or a further deposition of a metallic layer above the ITO-SAM structure was not evaluated.

The electrodeposition of metallic alloys on SAM-modified semiconducting substrates has not been accurately studied. These studies present high interest in order to confer magnetic or electronic properties for the development of devices for electronics ${ }^{44}$ and spintronics ${ }^{45}$. Along with this, the formation of a strongly bonded SAMs on a semiconducting surface, if allowing the formation of metallic thin films on it, would hamper diffusion of metallic layer constituents in the $\mathrm{SiO} 2$ semiconductor substrate $^{46}$. Moreover, thiol SAM-ITO substrates have been proposed to improve compactness and growth rate of metallic layers with respect to bare $\mathrm{ITO}^{47}$, as well as to inhibit $\mathrm{Ni}$ electroless deposition over $\mathrm{Pd} \operatorname{seeds}^{48}$. Thus, the electrodeposition of metallic layers, either ferromagnetic or non-magnetic, over alkanethiol-modified ITO substrates, deserves further investigation.

In this report we analyse the electrochemical preparation of metallic deposits of $\mathrm{CoNi}$ or $\mathrm{Pt}-\mathrm{CoNi}$ composite on an alkanethiol SAM-modified ITO substrate. The C12-SH SAM formed by immersion over a commercial ITO substrate is analysed by means of X-ray photoelectron spectroscopy (XPS), atomic force microscopy (AFM), electrochemical probe and contact angle measurements. The optimization of the incubation time, C12-SH solution concentration and ITO pretreatment are demonstrated to be critical for the compactness of the SAM. Electrodeposition of CoNi and Pt/CoNi on the ITO-SAM structure is studied in order to obtain non-magnetic or ferromagnetic hybrid structures. Surface morphology and magnetic response of the ITO-SAM-CoNi structure is evaluated by SEM and SQUID measurements. Although the electrodeposition process of the magnetic alloy is drastically hindered by the presence of the SAM layer, electron exchange can start through SAM surface defects leading to a continuous metallic layer on the SAM surface electrically connected to the ITO substrate by means of nanometric columns, probably formed by the accepted mushroom growth mechanism proposed for the electrodeposition on metallic substrates as gold.

\section{Experimental}

\subsection{SAM solutions}


1-dodecanethiol (C12-SH) (Merck, $\geq 98 \%$, synth. grade) was used as received. $5 \mathrm{mM}$ and $50 \mathrm{mM}$ thiol solutions were prepared with absolute ethanol (Panreac, UV-IR-HPLC PAI) and preserved at $5^{\circ} \mathrm{C}$ prior to use.

\subsection{Substrate pretreatment and SAM preparation}

ITO-coated $(25 \mathrm{~nm})$ glass $(2 \mathrm{~mm})$ substrates were pre-treated by means of ultrasonication in ultrapure water and a subsequent immersion in freshly prepared saturated $\mathrm{NaOH}$ solution (Merck, pellets, analytical grade) and pure $\mathrm{H}_{2} \mathrm{SO}_{4}$ (Acros Organics, 96\%). The freshly pre-treated ITO substrates were immersed in 5mM C12-SH, 50mM C12-SH or in pure C12-SH for different time at room temperature in a hermetically closed opaque glass vial. After incubation, samples were carefully rinsed in copious amounts of absolute ethanol and ultrapure water to remove physisorbed thiols, and blown dry with $\mathrm{N}_{2}$.

\subsection{SAM characterization}

AFM measurements were performed in a AFM Nanotech Microscope (Nanotech Electrónica S.L., Cervantes AFM system) in tapping mode using a $4.6 \mu \mathrm{m}, 45 \mu \mathrm{m}, 160 \mu \mathrm{m}$ (thickness, width, length) Arrow-NCR-20 monolithic, highly doped silicon tip (Nanoworld). Analysis of the topographical images (mean surface roughness) was performed by means of the WSxM 5.0 software $^{49}$. XPS measurements were carried out in a PHI ESCA-5500 Multitechnique System (Physical Electronics), with a monochromatic X-ray source (Al $\mathrm{K} \alpha$ line of $1486.6 \mathrm{eV}$ energy and $350 \mathrm{~W}$ ), placed perpendicular to the analyzer axis and calibrated using the $\mathrm{Ag} 3 d_{5 / 2}$ line with a full width at half maximum (FWHM) of $0.8 \mathrm{eV}$. The analysed area was a circle of $0.8 \mathrm{~mm}$ diameter, and the selected resolution for the spectra was $187.85 \mathrm{eV}$ of pass energy and $0.8 \mathrm{eV} / \mathrm{step}$ for the general spectra. For high-resolution spectra of the elements, $58.7 \mathrm{eV}$ of pass energy and $0.25 \mathrm{eV} / \mathrm{step}$ were used as XPS acquisition parameters. All measurements were made in an ultra-high vacuum (UHV) chamber, with a pressure between $5 \cdot 10^{-9}$ and $2 \cdot 10^{-8}$ torr. Angle-resolved XPS (AR-XPS) measurements were performed with the experimental conditions stated previously, but varying the angle between the surface and analyser from $15^{\circ}$ to $90^{\circ}$. CoNi deposits were etched by means of argon ions sputtering up to few nm prior to AR-XPS acquisition. Multipak 8.2 software was used for digital acquisition and peak deconvolution (single Gaussian curves fit), and all spectra were calibrated by setting the $\mathrm{C} 1 s \mathrm{C}$ $\mathrm{C} / \mathrm{C}-\mathrm{H}$ component at $285 \mathrm{eV}$. Contact angle measurements $(5 \mu 1$ ultrapure water drop, $15 \mathrm{sec}$. wait before image acquisition) were performed in a goniometer equipped with a Logitech CCD camera and Dropsnake analysis software. Electrochemical probe measurements were performed in a $2 \mathrm{mM}$ $\mathrm{Fe}\left(\mathrm{CN}_{6}\right)^{3-} / \mathrm{Fe}\left(\mathrm{CN}_{6}\right)^{4-}$ solution containing a $0.2 \mathrm{M} \mathrm{KNO}_{3}$ supporting electrolyte.

\subsection{Electrochemical measurements}


A customized 3-electrode electrochemical cell comprising a Pt spiral counter electrode, a double junction reference electrode (Metrohm, $\mathrm{Ag} / \mathrm{AgCl} / \mathrm{KCl} 3 \mathrm{M}$ ) and a SAM-modified or bare ITO substrate was used. A microcomputer-controlled PGSTAT30 or PGSTAT12 potentiostat/galvanostat with GPES software was used. Prior to every essay, the Pt counter electrode was flame annealed to light red and quenched in ultrapure water, and the electrolytic bath was de-aerated $15 \mathrm{~min}$ by Ar bubbling.

\subsection{Electrochemical deposition}

Pt deposits were obtained in a $5 \mathrm{mM} \mathrm{Na}_{2} \mathrm{PtCl}_{6}$ aqueous solution (Alfa Aesar) by applying a potential $\mathrm{E}=-0.32 \mathrm{~V}$ (vs. $\mathrm{Ag} / \mathrm{AgCl}$ ). $\mathrm{CoNi}$ deposits were carried out in a $0.2 \mathrm{M} \mathrm{CoCl} 2$ (Panreac), $0.9 \mathrm{M} \mathrm{NiCl}_{2}$ (Merck), $0.5 \mathrm{M} \mathrm{H}_{3} \mathrm{BO}_{3}$ (Merck) and $0.7 \mathrm{~g} \cdot 1^{-1}$ sodium saccharin (Merck) electrolytic bath, adjusted at $\mathrm{pH}=3$. Sodium saccharin is a well-known additive that allows minimizing electrodeposit internal strain during growth. All reagents used have analytical grade purity and are used as received. Aqueous solutions were prepared with ultrapure water $(18.2 \mathrm{M} \Omega \cdot \mathrm{cm})$ from a Millipore Gradient A10 UV purification system (Millipore). Deposits composition was determined by means of a X-ray Fluorescence Fisherscope system XDAL controlled with WinFTM software. A JEOL JSM-6510 equipment was used for the acquisition of FE-SEM micrographs, at a $15 \mathrm{keV}$ working voltage. Film thickness measurements were carried out in a Leica DCM 3D interferometer; data analysis was performed with the SensoScan DCM3D 3.1 software. Magnetic properties of the prepared structures were characterized using a SQUID magnetometer at $300 \mathrm{~K}$ in helium atmosphere. Measurements were performed under the application of a magnetic field in both perpendicular to plane and in-plane directions.

\section{Results and discussion}

\subsection{Evaluation of ITO pretreatment}

Substrate cleanliness is reported to play a critical role in the formation of high-compactness $\mathrm{SAMs}^{50}$. In gold electrodes, aggressive methods such as mechanical polishing, immersion in piranha solution or flame annealing result in a residue-free, high-quality surface feasible to further modification by a $\mathrm{SAM}^{51}$. However, these treatments cannot be used with ITO as they would compromise its surface integrity. Alternative pre-treatment methods, such as $\mathrm{UV} /$ ozone, $\mathrm{UV} / \mathrm{H}_{2} \mathrm{SO}_{4}$ or $\mathrm{UV} / \mathrm{NaOH}$ have been proposed but, despite achieving efficient contaminants removal, they imply the use of high-cost equipment. As a consequence, recent studies have focused on developing alternative procedures. Clark et al. studied the viability of a solvent-based cleaning protocol under proper solvent polarity election and sequential ordering ${ }^{52}$, and Karsi et al. suggested an ultrasonication (ultras.) degreasing step 
followed by a basic-acid media treatment ${ }^{43}$. The results of using these procedures in our ITO commercial samples, monitored by contact-angle measurements, are summarized in Table 1. It is noticeable the irreproducibility of the solvent-based treatments proposed by Clark et al., as they yield contact angles much higher than the value expected $\left(\theta_{\mathrm{th}} \sim 30^{\circ}\right)^{52}$. On the other hand, the basic-acid media treatment manages to obtain a highly-hydrophilic surface $\left(\theta_{\mathrm{f}}<5^{\circ}\right)$, in agreement with the theoretical value reported for this treatment $\left(\theta_{\mathrm{th}}<5^{\circ}\right)^{43}$, that guarantees successful and reproducible removal of both physisorbed inorganic and organic impurities. As a result, all ITO samples will be pretreated by this procedure prior to SAM formation.

\begin{tabular}{|c|c|c|c|}
\hline ITO pre-treatment method & $\theta_{\mathrm{o}} /{ }^{\mathrm{o}}$ & $\theta_{\mathrm{f}} /{ }^{\mathrm{o}}$ & $\theta_{\text {th }} /{ }^{\mathrm{o}}$ \\
\hline Detergent solution (5\%)+ ultrapure water + hexane + & 80 & 64 & 28 \\
isopropanol + acetone + methanol (20 min ultras. per step) & & & \\
\hline Detergent solution (5\%)+ ultrapure water + acetone + & 80 & 56 & 34 \\
isopropanol (20 min ultras. per step) & & & \\
\hline Acetone + isopropanol (20 min ultras. per step) & 80 & 63 & 56 \\
\hline Ultrapure water sonication + saturated NaOH + pure $\mathrm{H}_{2} \mathrm{SO}_{4}$ & 80 & $<5$ & $<5$ \\
\hline
\end{tabular}

Table 1. Evaluation of ITO pre-treatment methods as a function of the initial $\left(\theta_{\mathrm{o}}\right)$, final $\left(\theta_{\mathrm{f}}\right)$ and theoretical $\left(\theta_{\mathrm{th}}\right)$ contact angle in water.

\subsection{C12-SH SAM preparation}

\subsubsection{Adsorption kinetics: contact angle measurements}

Contact angle measurements are excellent qualitative criteria for evaluating surface hydrophobicity variation. The study of the adsorption kinetics of C12-SH over pre-treated ITO substrates, shown in Fig. 1A, reveals the influence of both incubation time and $\mathrm{C} 12-\mathrm{SH}$ solution concentration in the compactness of the SAM. At initial incubation stages (less than $5 \mathrm{~min}$ ), contact angles below $70^{\circ}$ inform that $\mathrm{C} 12-\mathrm{SH}$ molecules chemisorption kinetics has not reached its saturation rate. As a consequence, the resulting SAM is expected to exhibit an inhomogeneous, short-range orientational order due to its low compactness. At longer incubation times, effective SAM reorganization along the surface and further $\mathrm{C} 12-\mathrm{SH}$ molecules chemisorption occurs, allowing the formation of a more densely packed structure. This is supported by the fact that the maximum contact angle stabilizes at $\theta \sim 100^{\circ}$, independently of the C12-SH solution concentration and incubation time. This value is similar to those attributed to a densely packed C12-SH SAM on $\mathrm{ITO}^{43}$, initially confirming the capability of forming 
an alkanethiol monolayer over ITO by incubation either in ethanol solutions $(5 \mathrm{mM}$ or $50 \mathrm{mM})$ or in neat liquid.

\subsubsection{XPS analysis}

The interaction involved in the formation of a C12-SH SAM over ITO was elucidated by means of XPS measurements. The high-resolution XPS spectrum analysis of the sulphur S $2 p$ region, shown in Fig. 1 (B,C,D) was performed on ITO samples immersed in $5 \mathrm{mM} \mathrm{C12-SH}$ and pure C12-SH. The 5mM C12-SH sample signal can be decomposed into two components, with binding energies of 162.3 and $168.5 \mathrm{eV}$, respectively. The first one is ascribed to the $\mathrm{S} 2 p_{3 / 2}: \mathrm{S} 2 p_{1 / 2}$ doublet with a $2: 1$ intensity ratio characteristic of a thiolate group, confirming the formation of a covalent bond between the C12$\mathrm{SH}$ sulphur group and the ITO surface. This value, $\sim 0.4 \mathrm{eV}$ higher than that of an In-S or Sn-S bond on pure metal plates ${ }^{42}$, is attributed to the ITO surface composition and, in particular, the influence of the oxide species present. The $\mathrm{S} 2 p_{1 / 2}$ component, with an expected binding energy of $163.5 \mathrm{eV}$, is not well-resolved in the spectrum due to low sulphur S2p signal intensity and, as a consequence, its deconvolution is not possible. The second component, ascribed to highly oxidized sulphur (sulfate, sulfonate), can be related to the oxidation of unbounded thiol molecules located at the SAM surface by the X-ray irradiation during spectrum acquisition ${ }^{43}$ or to the presence impurities of oxidized sulphur species bonded to the ITO surface. Sulphur peaks broadening and appearance of additional XPS signals have already been reported for alkanethiol SAMs due to X-ray secondary electrons damage upon long X-ray time exposures ${ }^{53}$. The pure C12-SH sample signal can be decomposed into two complex components. The first component comprises a doublet, with binding energies of 163.5 and $164.7 \mathrm{eV}$. It is attributed again to the $\mathrm{S} 2 p_{3 / 2}: \mathrm{S} 2 p_{1 / 2}$ thiolate group doublet with intensity ratio $2: 1$. The second component comprises two peaks with binding energies of 169 and $170.3 \mathrm{eV}$. Unlike the 5mM C12-SH sample, more than one oxidized thiol species seems to contribute to the observed XPS signal. The XPS analysis allows detecting chemisorption of C12-SH molecules to ITO by means of a covalent bonding between the thiolate end group and In or Sn. However, other highly oxidized sulphur species originate the second component, as the manner that a densely-packed SAM layer cannot be formed.

\subsubsection{AFM measurements}

ITO topography imaging before and after the adsorption of a C12-SH layer was performed by means of tapping-mode AFM. Prior to this, the analysis of the topography of the ITO before (Fig. 1E) and after (Fig. 1F) undergoing the basic-acid pretreatment was performed, by evaluating the influence of the pretreatment in the surface root mean square roughness (RMS). Only a slight modification in RMS was observed ( $1 \mathrm{~nm}$ prior to pretreatment and $3 \mathrm{~nm}$ after pretreatment); in both cases usual characteristic holes at the ITO surface were observed. As a consequence, despite of its aggressive 
nature, the selected pretreatment is compatible with the formation of alkanethiol SAMs on ITO. The presence of holes (50 to $200 \mathrm{~nm}$ ) in the ITO can create non-conductive pinholes in the semiconducting layer. RMS values and surface topography after incubation of the pre-treated ITO substrates in a $5 \mathrm{mM}$ C12-SH solution (Fig. 1G) or in neat C12-SH (Fig. 1H) revealed an effective smoothing of the ITO surface as well as a significant masking of the ITO characteristic surface pattern. Both issues would initially confirm that, upon incubation in both cases, the C12-SH SAM is formed over ITO. Moreover, the filling of the aforementioned holes might respond to either a multilayer of physisorbed C12-SH molecules (if holes depth reaches glass surface) or to a monolayer of chemisorbed C12-SH molecules accompanied by physisorbed C12-SH molecules at their surface (if holes depth is inferior to $25 \mathrm{~nm}$ ). The presence of physisorbed molecules at the holes might explain the XPS S $2 p$ signals observed at higher binding energies, ascribed in this report to the in-situ oxidation of unbounded thiol molecules or to the presence of oxidized thiol impurities. The removal of these molecules is expected to be hampered by the difficulty of the solvent to penetrate the nanometric holes.

\subsubsection{Electrochemical response on ITO-SAM substrates}

In order to test the compactness of the SAM, the voltammetric response of a test electrochemical process (electrochemical probe) on the ITO-SAM substrate was performed. The charge transfer is intrinsically dependent on both the nature of the electrode surface as well as the structure of the electrical double-layer ${ }^{54}$. The presence of a SAM (dielectric nature) on an electrode hinders the electron transfer either by an effective reduction of the electrode electroactive area or by impeding the approach of the redox species to its surface. The $\mathrm{Fe}\left(\mathrm{CN}_{6}\right)^{3-} / \mathrm{Fe}\left(\mathrm{CN}_{6}\right)^{4-}$ redox pair is a standard probe because it implies a reversible, one-electron, outer-sphere redox reaction $\left(\mathrm{E}=0.153 \mathrm{~V}, k=0.03 \mathrm{~cm} \cdot \mathrm{s}^{-1}\right)^{55}$. If the compactness of the SAM is high, the redox pair $\mathrm{Fe}\left(\mathrm{CN}_{6}\right)^{3-} / \mathrm{Fe}\left(\mathrm{CN}_{6}\right)^{4-}$ will not manage to get in direct contact with the ITO surface, leading to a substantial decay in the electrochemical current density of the redox processes involved. If the compactness of the SAM is low, the electroactive species will manage to perform electron transfer processes through SAM surface defects, observing a non-negligible current density.

Figure 2 shows the cyclic voltammograms obtained in a $2 \mathrm{mM} \mathrm{Fe}\left(\mathrm{CN}_{6}\right)^{3-} / \mathrm{Fe}\left(\mathrm{CN}_{6}\right)^{4-}$ solution for the C12-SH SAM modified ITO electrodes at different times of incubation in 5mM or pure C12-SH. For the $5 \mathrm{mM}$ C12-SH incubated ITO samples, a significant decay of the current density was observed at 2 $\mathrm{h}$ of incubation, but a similar electrochemical response was obtained for $5 \mathrm{~h}$ of incubation, which reveals that a limit in the coverage of the ITO was attained and that, accordingly, a densely-packed SAM was not formed in these conditions. The presence of a low compact C12-SH layer allows the approaching of the electroactive species (through the SAM defects) to the ITO electrode and the 
subsequent electron transfer. When longer inmersion times were tested, the electrochemical response of the redox probe increased, indicating the presence of an even less compact SAM. This could be attributed to the fact that, in an ethanol media, a dynamic competitive adsorption process could occur. As the interaction of alkanethiols with ITO is expected to be weaker than to a gold surface, ethanol molecules may replace C12-SH adsorbed molecules at the ITO surface upon long modification times, leading to a decrease in the SAM compactness. A similar phenomenon was reported for silane-based $\mathrm{SAMs}^{56}$, for which upon substrate rinsing, a SAM unpacking process occurred that could not be reversed upon re-immersion in the SAM solution. The former phenomenon would be in agreement with the results obtained by Obeng and Bard for the adsorption of the complex [Ru(bpy) 2 (4-methyl-4'(dodecyl-1-thiol)-2,2'-bipyridine) $]^{2+}$ over commercial ITO by immersion in $2 \mathrm{mM}$ solutions in dichloromethane ${ }^{57}$. Repeated washing of the ITO-modified samples with dichloromethane, methanol or ethanol can produce some competitive replacement of the thiol molecules with solvent molecules at the ITO surface. Kondo et al. observed also that, upon preparation of ferrocenylalkane thiol SAMs over ITO upon incubation in $1 \mathrm{mM}$ solutions at $50^{\circ} \mathrm{C}$ for between 10 and 40 hours, both maximum surface concentration $\Gamma_{\max }$ and adsorption rate constant $k_{\text {ads }}$ were much smaller than with respect to the $\mathrm{Au}$ substrate despite using higher temperatures and thiol solution concentrations ${ }^{58}$. This was adduced to the weak interaction of the adsorbed thiol molecules to ITO in this concentration range, which may lead only to partial surface coverages of the substrate. As a consequence, the electrochemical probe analysis leads to the conclusion that both contact angle and AFM topographies of samples prepared by this methodology only deliver qualitative information about SAM compactness. Hence, electrochemical characterization of the SAM should be always performed to estimate its compactness, especially in the case of posterior electrodeposition.

In the case of ITO samples incubated in pure C12-SH, a more significant decay of the current density was observed, and a quasi-stationary voltammetric profile was obtained from 5-6 hours of incubation onwards. In these conditions, the maximum coverage was attained, but the non-negligible current density detected informs of the presence of defects in the SAM. However, in order to prepare the metallic deposits over the ITO-SAM structures, an incubation time of 5 hours in pure thiol was selected to minimize pinhole presence at the SAM and, consequently, to ensure preferential electrodeposition of the metallic layer on the top of the SAM.

Once the experimental conditions have been optimised, the electrochemical stability of the ITO-SAM structure was analysed by recording cyclic voltammogram in an aggressive medium $\left(\mathrm{H}_{2} \mathrm{SO}_{4} 0.05 \mathrm{M}\right)$. Both ITO and ITO-SAM exhibit in the $-0.5 /+1.5 \mathrm{~V}$ potential range only redox processes concerning the solvent (massive hydrogen evolution or oxygen formation) (Fig. 2C). This issue shows the wide electrochemical window exhibited by both samples, confirming the stability of the ITO-SAM system 
in acidic media that allows further metal electrodeposition. Moreover, the ITO-SAM sample exhibits a significant displacement of redox processes to higher absolute potentials values as well as a lower interfacial capacitance $\left(2.5 \mu \mathrm{F} \cdot \mathrm{cm}^{-2}\right)$ with respect to bare ITO $\left(6.25 \mu \mathrm{F} \cdot \mathrm{cm}^{-2}\right)$ in the $0.9-1.1 \mathrm{~V}$ voltage range, as a consequence of the organic layer adsorption. Theoretical interfacial capacitance $\left(\mathrm{C}_{\mathrm{th}}\right)$ value for a C12-SH monolayer (assuming dielectric constant $\varepsilon=2.5$, molecular length $\mathrm{d}=17.6 \AA$, and tilt angle with respect to normal surface axis $\left.\alpha=0^{\circ}\right)^{59,60}$ is $1.3 \mu \mathrm{F} \cdot \mathrm{cm}^{-2}$. Differences observed between both theoretical and experimental values can be ascribed to deviations with respect to the parallel plate model assumed for calculating $\mathrm{C}_{\text {th }}$ and to the presence of defects in the SAM. A more tilted conformation of C12-SH molecules present in a SAM with surface defects with respect to a denselypacked C12-SH SAM leads to an overestimation of the distance between the parallel plates, i. e. the electrical double layer thickness, consequently leading to higher interfacial capacitance values.

\subsection{Electrodeposition of a ferromagnetic CoNi alloy over ITO-SAM: electrochemical study}

The cyclic voltammograms of ITO and ITO-SAM substrates in an electrolytic bath containing Co(II) and $\mathrm{Ni}(\mathrm{II})$ will allow us to determine whether electrodeposition is possible over these substrates and if this deposition leads to the formation of a metallic alloy. The voltammetric profile obtained over ITO is similar to that obtained in other substrates such as vitreous carbon, silicon, $\mathrm{Si} / \mathrm{Ti} / \mathrm{Ni}$ or $\mathrm{Si} / \mathrm{Cr} / \mathrm{Cu}^{61}$, and the deposition process starts at approx. $-750 \mathrm{mV}$ (Fig. 3, curve a). Over the ITO-SAM structure, deposition current is observed only at more negative potentials $(E=-850 \mathrm{mV})$ and the process is drastically slower (Fig. 3, curve b), as predicted by electrochemical probe results. The presence of the C12-SH SAM hinders significantly the electron transfer kinetics, requiring higher cathodic potential values to permit the deposition process. Moreover, for both redox processes (deposition and alloy oxidation), the $\mathrm{j} / \mathrm{E}$ slopes are substantially lower for the ITO-SAM. Upon inversion of the scanning sweep, the nucleation and growth loop characteristic of electrodeposition processes is observed. This issue confirms the viability of electrodeposition over SAM-modified ITO samples, although very hindered by the presence of the SAM. The detection of a single oxidation peak in the anodic region is in good agreement with the formation of a CoNi metallic alloy. The high value of the $\mathrm{Q}_{\mathrm{ox}} / \mathrm{Q}_{\text {red }}$ ratio obtained for bare ITO (0.96) and for ITO-SAM sample (0.89) in the potential range applied (-0.75/-1.2 $\mathrm{V}$ and $-0.85 /-1.4 \mathrm{~V}$, respectively) informs of the low contribution of hydrogen evolution during the deposition process. To favour the electrical contact with the ITO-SAM samples, a colloidal Ag layer was applied to the end of the ITO-coated side of the sample that connects the working electrode (WE) to the electrochemical cell. The cyclic voltammogram of the colloidal Ag-coated ITO-SAM sample in the $\mathrm{Co}(\mathrm{II}) / \mathrm{Ni}$ (II) electrolytic bath (Fig. 3, curve c) exhibits a significant increase in the $\mathrm{j} / \mathrm{E}$ slope in comparison to the non-coated sample, resulting in a two-fold increase of the deposition current density 
at $-1.4 \mathrm{~V}$. Thus, all electrochemical deposits over ITO-SAM samples will be prepared according to this methodology to increase the deposition rate.

The potentiostatic electrodeposition of the CoNi alloy was tested, at two deposition rates, on both ITO and ITO-SAM substrates. In each case the deposition potentials were adjusted to attain similar $\mathrm{j}$ - $\mathrm{t}$ profiles on ITO and ITO-SAM, in order to compare the deposits obtained over the two substrates at similar deposition rate. Over the ITO-SAM substrate, more negative potentials were applied to obtain the same j-t profile than over bare ITO, due to the blocking effect of the SAM in the electron transfer process.

The deposition at the more negative potentials (-0.94 V for ITO, -1.1 V for ITO-SAM) (curves $g$ and $\mathrm{f}$ in Figure 4), selected to achieve growth rates similar to those obtained for CoNi deposits on $\mathrm{Si} / \mathrm{Ti} / \mathrm{Au}$ substrates ${ }^{61}$ leads to incoherent, low adherence deposits with micrometric cracks. However, when the deposition process is slower $(0.78 \mathrm{~V}$ for ITO, $-0.92 \mathrm{~V}$ for ITO-SAM) (curves b and $\mathrm{d}$ in Figure 4) uniform, non-cracked deposits were obtained. The FE-SEM micrographs (Figs. 4B and 4C) show that both deposits present a thin-grain, nodular morphology, whose grain size cannot be estimated quantitatively due to the technique resolution limits, this commonly observed with some CoNi deposits $^{62}$. As similar stationary current density (approx. $-2 \mathrm{~mA} \cdot \mathrm{cm}^{-2}$ ), corresponding to a similar deposition rate, was achieved for the two substrates, similar composition (74 wt \% Co), morphology and thickness $\left(270 \mathrm{~nm}\right.$ for deposition at a charge density $<_{\sigma_{\mathrm{q}}}>=-1 \mathrm{C} \cdot \mathrm{cm}^{-2}$ over ITO, $250 \mathrm{~nm}$ for deposition at a charge density $<\sigma_{\mathrm{q}}>=-0.7 \mathrm{C} \cdot \mathrm{cm}^{-2}$ over ITO-SAM) of the CoNi films is obtained. Thus, similar deposition rates, obtained by careful election of deposition potentials, lead to equivalent morphologies and compositions of the resultant deposits in ITO as well as ITO-SAM. If CoNi electrodeposition is performed over ITO-SAM with the applied potential optimised for ITO, the chronoamperometric profile $(E=-0.78 \mathrm{~V}$, Fig. 4, curve a) confirms the significant hindrance of the SAM in the electron transfer process. Deposition of CoNi layers is, therefore, possible on the ITOSAM substrates: the expected initial growth of the deposits through the SAM defects evolves to a metallic fine grained layer of CoNi covering the sample, probably according to a mushroom growth mechanism accepted for SAM-modified metallic substrates ${ }^{30,31}$.

The Co-rich deposits obtained despite of using a Ni-rich electrolytic bath (Co:Ni molar ratio in solution being 1:4.5) corroborate that the anomalous codeposition mechanism of the CoNi deposition in the selected bath ${ }^{63}$ is maintained also over ITO-SAM substrates. In this report, we have selected a long-stablished, optimized bath with a Co:Ni molar ratio capable of forming continuous, thin-grain and non-strained deposits on a wide range of substrates ${ }^{61,63}$. However, the modification of the Co(II)/Ni(II) 
molar ratio in the electrolytic bath would allow to tailor the CoNi deposit composition of the CoNi layers obtained over the ITO-SAM structures.

Figs. 5A and 5B show the XPS spectra analysis of a pre-etched CoNi alloy thin film (see Experimental) deposited over an ITO-SAM sample in the region of $\mathrm{Co} 2 p_{3 / 2}$ and $\mathrm{Ni} 2 p_{3 / 2}$, respectively. The Co $2 p_{3 / 2}$ signal can be decomposed in three components with binding energies of 781.2, 783.9 and $785.9 \mathrm{eV}$. The first one, ascribed to a CoNi alloy phase, has the largest photoemission intensity of the deconvoluted signals, acknowledging the high efficacy and selectivity of the direct electrodeposition methodology for preparing alloy metallic layers. The second and third component are assigned to trace amounts of oxidised Co species, those being $\mathrm{Co}_{3} \mathrm{O}_{4}$ and $\mathrm{Co}(\mathrm{OH})_{2}$, which reveals some partial oxidation of the deposits either prior to or during XPS spectra acquisition. The Ni2 $p_{3 / 2}$ is similarly deconvoluted in three components with binding energies of 856.2, 859.2 and 861.9. The first component is again assigned to the CoNi alloy phase, and the two remaining components to trace amounts of Ni species as $\mathrm{NiO}$ and $\mathrm{Ni}(\mathrm{OH})_{2}$. All the element-specific core level CoNi emissions reported in this essay are shifted approx. $3 \mathrm{eV}$ to higher binding energies in contrast to the values reported by Kim et. al over a silicon surface $^{64}$. The origin of this shift relies in the junction of a highly conductive CoNi alloy film with a low conductive ITO-SAM surface that difficult a proper XPS spectra calibration with respect to the $\mathrm{C} 1 s \mathrm{C}-\mathrm{C} / \mathrm{C}-\mathrm{H}$ component, as well as the different nature of the substrate where CoNi is deposited.

AR-XPS measurements were performed to identify where the CoNi layer is mainly present in the ITOSAM structure. Figs. 5C and 5D show the relative intensity ratios of the $\operatorname{In} 3 d_{5 / 2}$ peak with respect to the C) $\mathrm{Co} 2 p_{3 / 2}$ and D) $\mathrm{Ni} 2 p_{3 / 2}$ peaks ascribed to the CoNi alloy phase of a CoNi electrodeposit prepared over ITO-SAM as a function of the XPS take-off angle $\alpha$ (angle between the sample surface plane and the direction of the photoelectrons detected by the analyser). The intensity ratios of the analysed elements are normalized with respect to the core-level emission values obtained at $\alpha=90^{\circ}$.

Upon the application of a X-ray incident beam, the scape depth of the photoelectrons emitted from the sample is highly dependent on $\alpha$. Nearly grazing X-ray incidence (low $\alpha$ values) measurements are sensitive to the more superficial atomic/molecular layers, in this case the surface of the SAM, due to the lower scape depth (kinetic energy) of the photoemitted electrons detected. Perpendicular X-ray incidence (high $\alpha$ values) measurements are more sensitive to the less superficial atomic/molecular layers, in this case to the ITO surface, due to higher scape depth of the photoemitted electrons detected. Thus, CoNi deposit is mainly accumulated at the top of the SAM should lead to higher photoemission intensities of $\mathrm{CoNi}$ at low $\alpha$ than at high $\alpha$. When going to X-ray normal incidence $\left(\alpha=90^{\circ}\right)$ to nearly grazing incidence $\left(\alpha=15^{\circ}\right)$, a steep decay in the $\operatorname{In} 3 d_{5 / 2} / \mathrm{Co} 2 p_{3 / 2}$ and $\operatorname{In} 3 d_{5 / 2} / \mathrm{Ni} 2 p_{3 / 2}$ 
normalized relative intensity ratios is observed, indicating that the CoNi metallic film is mainly located on top of the C12-SH SAM, in agreement with the mushroom-type growth mechanism.

The magnetic properties of the ITO-SAM-CoNi structures have been analysed and compared with the magnetic response of the ITO-CoNi samples, with the same nominal thickness of CoNi in both cases, in order to detect both the formation of a magnetic ITO-SAM-CoNI structure and detect the possible influence of the SAM presence. The typical normalized hysteresis curves $\left(\mathrm{M} / \mathrm{M}_{\mathrm{s}} \mathrm{vs}\right.$. H, being $\mathrm{M}$ the magnetization, $M_{s}$ the magnetization of saturation, and $H$ the magnetic field applied) of ferromagnetic materials have been observed in both cases (Figs. 5E and 5F). Due to the shape anisotropy of the films, consequence of their 2D nature, the samples are easily magnetized when the magnetic field is applied parallel to the film plane. Both CoNi deposits on ITO and ITO-SAM substrates present similar differences between the magnetic curves recorded in parallel or perpendicular configuration, which demonstrates that CoNi deposits over ITO-SAM are mainly accumulated on the SAM surface, leading to a mainly $2 \mathrm{D}$ layer, because the shape anisotropy is maintained. Moreover, the coercivity $\left(\mathrm{H}_{\mathrm{c}}\right)$ was low in both cases, as it is expected for the CoNi deposits ${ }^{65-67}$, showing the same thickness, composition and similar fine-grained morphology. However, zoomed curves reveal that the magnetic signal is different, which corroborates the formation of ITO-SAM-CoNi structures, different than the ITO-CoNi ones. The difference is the formation of a main CoNi layer over the SAM, but anchored or connected to the ITO substrate by means CoNi columns formed through the defects of the SAM.

3.4 Electrodeposition of CoNi over a preformed Pt nuclei layer: preparation of hybrid composite structures

Once we have confirmed the presence of the CoNi alloy deposit above a SAM, we proceeded to evaluate the viability of depositing other metallic elements or hybrid structures on ITO-SAM substrates. The architectural growth of hybrid structures over ITO-SAM samples could be easily performed upon tailoring both electrodeposition parameters and electrolytic bath composition. In this report, the electrodeposition of a CoNi alloy was evaluated on an ITO-SAM substrate modified with a Pt sublayer (ITO-SAM-Pt) obtained by electrodeposition $\left(\mathrm{E}=-0.32 \mathrm{~V}, \mathrm{t}=120\right.$ s) in a $5 \mathrm{mM} \mathrm{Na} \mathrm{PtCl}_{6}$ solution. Pt electrodeposition parameters were chosen to overcome C12-SH SAM inherent electric resistance and to favour the formation of a continuous layer, after voltammetric analysis of ITO-SAM in a $5 \mathrm{mM} \mathrm{Na}_{2} \mathrm{PtCl}_{6}$ solution (not shown). Figure $6 \mathrm{~A}$ shows that platinum deposition is indeed hindered by the presence of the SAM layer: the stationary current density detected in the chronoamperometric experiments at equivalent deposition conditions is drastically lower on ITO-SAM structures (-1.25 $\mathrm{mA} \cdot \mathrm{cm}^{-2}$, curve b) than on bare ITO $\left(-4.5 \mathrm{~mA} \cdot \mathrm{cm}^{-2}\right.$, curve a). The presence of platinum on the ITO- 
SAM substrates was detected by a cyclic voltammetry test in $0.05 \mathrm{M} \mathrm{H}_{2} \mathrm{SO}_{4}$ medium, which reveals the typical profile in which the superficial platinum oxidation from around $0.8 \mathrm{~V}$ and the corresponding reduction process (peak centered at $0.4 \mathrm{~V}$ ) are observed (Figure 6B). The resulting platinum deposit (theoretical thickness $=12 \mathrm{~nm}$ for 100\% deposition efficiency) over the ITO-SAM sample shows a large presence of thick-grain, nodular Pt nuclei despite the low charge density applied $\left(-0.05 \mathrm{C} \cdot \mathrm{cm}^{-2}\right)$ (Figure $6 \mathrm{C}$ ). The non-homogeneous size distribution of the observed grains (between 5 and $150 \mathrm{~nm}$ ) is characteristic of the mushroom-type growth mechanism ${ }^{30,31}$, confirming the initial nucleation and columnar growth of Pt at SAM surface defects and subsequent 3D growth at the SAM surface. The AR-XPS measurements also reveal preferential accumulation of platinum on the top of the C12-SAM. The Pt4f XPS spectrum of the Pt obtained by electrodeposition (Fig. 6D) can be decomposed in two components, with binding energies of 71.9 and $75.2 \mathrm{eV}$, corresponding to the corelevel emissions of Pt $4 f_{7 / 2}$ and $\mathrm{Pt} 4 f_{5 / 2}$, respectively. Binding energy values are positively shifted approx. $0.7 \mathrm{eV}$ in comparison to the binding energy values expected from a Pt (111) bulk clean surface ${ }^{68}$. This shift can also be adduced to the high-conductive Pt/low conductive C12-SH SAM junction present in the sample, previously observed by Uosaki et al. for Pt (111) over a dithiol SAM ${ }^{69}$. Figs. 6E and 6F show the AR-XPS relative intensity ratios of the E) In $3 d_{5 / 2}$ and F) S2 $p_{3 / 2}$ deconvoluted peaks with respect to the Pt $4 f_{7 / 2}$ peak, for the platinum on ITO-SAM structure, as a function of $\alpha$. The intensity ratios of the analysed elements are again normalized with respect to the core-level emission values obtained at $\alpha=90^{\circ}$. As occurred with the CoNi alloy, a steep decay in the $\operatorname{In} 3 d_{5 / 2} / \operatorname{Pt} 4 f_{7 / 2}$ and S $2 p_{3 / 2} /$ $\operatorname{Pt} 4 f_{7 / 2}$ normalized intensity ratios is observed upon going from X-ray normal incidence $\left(\alpha=90^{\circ}\right)$ to nearly grazing incidence $\left(\alpha=15^{\circ}\right)$. Uosaki demonstrated that, if both Pt deposit/dithiol SAM and Pt deposit/Au substrate normalized XPS intensity ratios decayed with higher $\alpha$ values, the Pt deposit was preferentially obtained above the $\mathrm{SAM}^{69}$.

The presence of a highly-conductive Pt layer grown from the SAM defects but distributed preferentially on the top of the SAM is not only expected to ensure CoNi selective deposition above the SAM, but also to improve both growth rate and applied potential values. The potentionstatic electrodeposition of $\mathrm{CoNi}$ was initially performed over ITO-SAM-Pt at the electrodeposition conditions selected for the CoNi deposition above a SAM $\left(-0.92 \mathrm{~V},-1 \mathrm{C} \cdot \mathrm{cm}^{-2}\right)$. A higher stationary current density (approx. $-3.2 \mathrm{~mA} \cdot \mathrm{cm}^{-2}$ ) (Fig. 4, curve e) is achieved in comparison to the growth rate obtained without Pt, confirming the SAM surface conductivity enhancement produced by Pt presence, but in these conditions the adherence of the CoNi deposit is very low. The application of less negative potentials $(-0.88 \mathrm{~V})$ (Fig. 4, curve c) to attain similar stationary currents than for CoNi electrodeposition over ITO-SAM, allowed the preparation of a high-adherent CoNi deposit over ITOSAM-Pt. The FE-SEM micrograph of the resultant deposit (Fig. 7) shows an evident change in the 
CoNi deposit morphology. Thus, the presence of an underlying Pt layer not only forces additional electrodeposition optimisation but also alters the CoNi characteristic surface morphology. To sum up, the preparation of a Pt-CoNi hybrid composite structure mainly on the top of an ITO-SAM is also possible. Hence, this report widens the experimental techniques available for hybrid composite production over SAM-modified substrates, providing a low cost and environment-friendly method susceptible to be used for different hybrid structures, such as nanopatterning of soft-magnetic thin films over semiconducting substrates (ITO) using SAMs as templates.

\section{Conclusions}

Electrodeposition methods allow preparing magnetic layers over ITO-alkanethiol SAM substrates. XPS studies allow us to propose the chemisorption of the molecules interacting with the ITO surface. However, in order to assess quantitatively the formation of a partial or compact C12-SH layer covering the ITO surface, electrochemical probe experiments or cyclic voltammetries in blank solutions are necessary. A drastic cleaning of the ITO surface, a five-hour inmersion in pure $\mathrm{C} 12-\mathrm{SH}$, and a posterior severe removal of physisorbed molecules, are proposed as the best conditions to obtain $\mathrm{C} 12$ SH SAM over ITO.

The electrodeposition of CoNi alloy on SAM-modified ITO substrate is possible, although it is significantly hindered in the conditions selected to attain the maximum thiol adsorption. A metallic layer is formed on the SAM, which must be anchored to the ITO surface by nanocolumns grown through SAM surface defects according to the mushroom growth mechanism. Cracked or more continuous films, of fine-grained morphology, are obtained by controlling the deposition rate. The mechanism of the anomalous CoNi alloy co-deposition is maintained on the ITO-SAM substrates. The magnetic properties of the structures are influenced by the SAM presence. Pt can be also directly deposited, which can be used to promote modified CoNi electrodeposited films on the ITO-SAM substrates. The formation of magnetic structures as ITO-SAM-CoNi or ITO-SAM-Pt-CoNi with noncracked metallic films has been possible electrochemically by adjusting the deposition potentials and the deposition rate. This demonstrates the potentiality of electrochemical methods to contribute at the fabrication of hybrid structures in which semiconducting modified substrates can accept metallic magnetic layers anchored through the SAM. This can be the first stage of using the SAM surface defects as a support to nanopattern electrodeposited magnetic films over semiconductor substrates as ITO. 


\section{Acknowledgements}

Work supported by contract CQT2010-20726 from MINECO. Authors thank the CCiTUB for their equipment. D. E. would also like to thank the MIECD for its financial support (Collaboration Grant).

\section{References}

1. M. Galbiati, C. Barraud, S. Tatay, K. Bouzehouane, C. Deranlot, E. Jacquet, A. Fert, P. Seneor, R. Mattana and F. Petroff, Adv. Mater., 2012, 24, 6429-6432.

2. H. Fan and G. P. Lopez, Langmui, 1997, 13, 119-121.

3. A. C. Templeton et. al., J. Am. Chem. Soc., 1998, 120, 4845-4849.

4. G. J. Wegner, H. J. Lee and R. M. Corn, Anal. Chem., 2002, 74, 5161-5168.

5. M. Lestelius, B. Liedberg and P. Tengvall, Langmuir, 1997, 13, 5900-5908.

6. A. Lando, K. Lauwaet and P. Lievens, Phys. Chem. Chem. Phys., 2009, 11, 1521-1525.

7. G. Thakur, K. Prashanthi and T. Thundat, Sci. Rep., 2013, 3, 1923.

8. J. Zhao and K. Uosaki, Langmuir, 2001, 17, 7784-7788.

9. A. Ulman, Chem. Rev., 1996, 96, 1533-1554.

10. R.G. Nuzzo and D.L. Allara, J. Am. Chem. Soc., 1983, 105, 4481-4483.

11. T. Sum and K. Uosaki, J. Phys. Chem. B, 2004, 108, 6422-6428.

12. T. Doneux, R.J. Nichols and C. Buess-Herman, J. Electroanal. Chem., 2008, 621, 267-276.

13. E.H.J. Wong, G.L. May and C.P. Wilde, Electrochim. Acta, 2013, 109, 67-74.

14. J.M. Campiña, A. Martins and F. Silva, Electrochim. Acta, 2008, 53, 7681-7689.

15. V. Prucker, O. Rubio-Pons, M. Bockstedte, H. Wang, P.B. Coto and M. Thoss, J. Phys. Chem. C, 2013, 117, 25334-25342.

16. M. Yuan, S. Zhan, X. Zhou, Y. Liu, L. Feng, Y. Lin, Z. Zhang and J. Hu, Langmuir, 2008, 24, 8707-8710.

17. K. Heister, M. Zharnikov and M. Grunze, J. Phys. Chem. B, 2001, 105, 4058-4061.

18. R.P. Andres, T. Bein, M. Dorogi, S. Feng, J.I. Henderson, C.P. Kubiak, W. Mahoney, R.G. Osifchin and R. Reifenberger, Science, 1996, 272,1323-1325.

19. Y. Ge, T. Weidner, H. Ahn, J.E. Whitten and M. Zharnikov, J. Phys. Chem. C, 2009, 113, 4575 .

20. Y.L. Loo, R.L. Willet, K.W. Baldwin and J.A. Rogers, J. Am. Chem. Soc., 2002, 12, 76547655 .

21. Y.L. Loo, D.V. Lang, J.A. Rogers and J.W.P. Hsu, Nano Lett., 2003, 3, 913-917.

22. E. Menard, L. Bilhaut, J. Zaumseil and J.A. Rogers, Langmuir, 2004, 20, 6871-6878. 
23. D. Aldakov, Y. Bonnassieux, B. Geffroy and S. Palacin, ACS Appl. Mater. Interfaces, 2009, 3, 584-589.

24. B. Radha, G. Liu, D.J. Eichelsdoerfer, G.U. Kulkarni and C.A. Mirkin, ACS Nano, 2013, 3, 2602.

25. A. Serrà, E. Gómez, J.F. López-Barbera, J. Nogués and E. Vallés, ACS Nano, 2014, 5, 46304639.

26. M. A. Schneeweiss and D. M. Kolb, Phys. Stat. Sol., 1999, 173, 51-71.

27. H. Hagenström, M. A. Schneeweiss and D. M. Kolb, Electrochim. Acta, 1999, 45, 1141-1145.

28. H. Hagenström, M. J. Esplandiú and D. M. Kolb, Langmuir, 2001, 17, 839-848.

29. T. Baunach and D. M. Kolb, Anal. Bioanal. Chem., 2002, 373, 743-748.

30. O. Cavalleri, S. E. Gilbert and K. Kern, Chem. Phys. Lett., 1997, 269, 479-484.

31. T. Felgenhauer, C. Yan, W. Geyer, H.-T. Rong and A. Gölzhäuser, Appl. Phys. Lett., 2001, 79, 3323-3325.

32. P. L. Schilardi, O. Azzaroni and R. C. Salvarezza, Langmuir, 2001, 17, 2748-2752.

33. O. Azzaroni, P. L. Schilardi and R. C. Salvarezza, Appl. Phys. Lett., 2002, 80, 1061-1063.

34. O. Azzaroni, P. L. Schilardi and R. C. Salvarezza, Electrochim. Acta, 2003, 48, 3107-3114.

35. S. Nagaoka, K. Horiuchi, M. Shikishima and A. Nakajima, J. Phys. Chem C, 2011, 115, 2421524220.

36. S. Tatay, C. Barraud, M. Galbiati, P. Seneor, R. Mattana, K. Bouzehouane, C. Deranlot, E. Jacquet, A. Forment-Aliaga, P. Jegou, A. Fert and F. Petroff, ACS Nano, 2012, 10, 8753-8757.

37. M. Cortés, E. Gómez, J. Sadler and E. Vallés, Electrochim. Acta, 2011, 56, 4087-4091.

38. A.C.T. Alves, D.J.C. Gomes, J.R. Silva and G.B. Silva, Appl. Surf. Sci., 2013, 279, 67-70.

39. H. Kim, A. Piqué, J.S. Horwitz, H. Mattoussi, H. Murata, Z.H. Kafafi and D.B. Chrisey, Appl. Phys. Lett., 1999, 23, 3444-3446.

40. E. Sargent, Nat. Photonics, 2012, 6, 133-135.

41. C.G. Granqvist and A. Hultåker, Thin Solid Films, 2002, 411, 1-5.

42. C. Yan, M. Zharnikov, A. Gölzhäuser and M. Grunze, Langmuir, 2000, 16, 6208.

43. N. Karsi, P. Lang, M. Chehimi, M. Delamar and G. Horowitz, Langmuir, 2006, 22, 3118.

44. R. Haag, M. A. Rampi, R. E. Holmlin and G. M. Whitesides, J. Am. Chem. Soc., 1999, 121, 7895-7906.

45. F. Ay, B. Aktaş, R. I. Khaibullin, V. I. Nuzhdin and B. Z. Rameev, J. Magn. Magn. Mater., 2015, 375, 129-135.

46. E. Glickman, A. Inberg, N. Fishelson and Y. Shaham-Diamand, Microelectron. Eng., 2007, 84, 2466-2470.

47. C. Park, S. Seo, H. Shin, B. D. Sarwade, J. Na and E. Kim, Chem. Sci., 2015, 6, 596-602. 
48. A. Hovestad, H. Rendering and A. W. Maijenburg, J. Appl. Electrochem, 2012, 42, 753-761. 49. I. Horcas, R. Fernández, J. M. Gómez-Rodríguez, J. Colchero, J. Gómez-Herrero and A. M. Baro, Rev. Sci. Instrum., 2007, 78, 13705-13708.

50. J. Tkac and J.J. Davis, J. Electroanal. Chem., 2008, 621, 117-120.

51. G. Feng, T. Niu, X. You, Z. Wan, Q. Kong and S. Bi, Analyst, 2011, 136, 5058-5063.

52. M.D. Clark and B.J. Leever, Sol. Energ. Mat. Sol. C, 2013, 116, 270-274.

53. M. Zharnikov, W. Geyer, A. Gölzhäuser, S. Frey and M. Grunze, Phys. Chem. Chem. Phys., 1999, 1, 3163-3171.

54. D. García-Raya, R. Madueño, J.M. Sevilla, M. Blázquez and T. Pineda, Electrochim. Acta, 2008, 53, 8026-8033.

55. V. Marecek, Z. Samec and J. Weber, J. Electroanal. Chem, 1978, 94, 169-185.

56. A.G. Richter, C.J. Yu, A. Datta, J. Kmetko and P. Dutta, Phys. Rev. E, 2000, 61, 607-615.

57. Y. S. Obeng and A. J. Bard, Langmuir, 1991, 7, 195-201.

58. T. Kondo, M. Takechi, Y. Sato and K. Uosaki, J. Electroanal. Chem., 1995, 381, 203-209.

59. F. A. Aguiar, R. Campos, C. Wang, R. Jitchati, A. S. Batsanov, M. R. Bryce and R. Kataky, Phys. Chem. Chem. Phys., 2010, 12, 14804-14811.

60. Y. Yang, in Introduction to Organic Electronic and Optoelectronic Materials and Devices, ed.

S.-S. Sun and L. R. Dalton, CRC Press, Boca Raton, 1st edn., 2008, ch. 22, p. 711.

61. J. Vilana, E. Gómez and E. Vallés, J. Electroanal. Chem., 2013, 703, 88-96.

62. X. Tarrús, M. Montiel, E. Vallés and E. Gómez, Int. J. Hydrogen Energ., 2014, 39, 6705-6713.

63. E. Gómez, J. Ramírez and E. Vallés, J. Appl. Electrochem.1998, 28, 71-79.

64. D. Kim, R. Shanmugam, M.-R. Choi and B. Yoo, Electrochim. Acta, 2012, 75, 42-48.

65. P. Cojocaru, L. Magganin, E. Gómez, E. Vallés, F. Liu, C. Carraro and R. Maboudian, J. Microtech. Microeng., 2010, 20, 125017.

66. E. Gómez, S. Pané and E. Vallés, Electrochim. Acta, 2005, 51, 146-153.

67. E. Gómez, S. Pané and E. Vallés, Electrochem. Commun., 2005, 7, 1225-1231.

68. C.R. Parkinson, M. Walker and C.F. McConville, Surf. Sci., 2003, 545, 19-33.

69. D. Qu and K. Uosaki, J. Electroanal. Chem., 2011, 662, 80-86. 

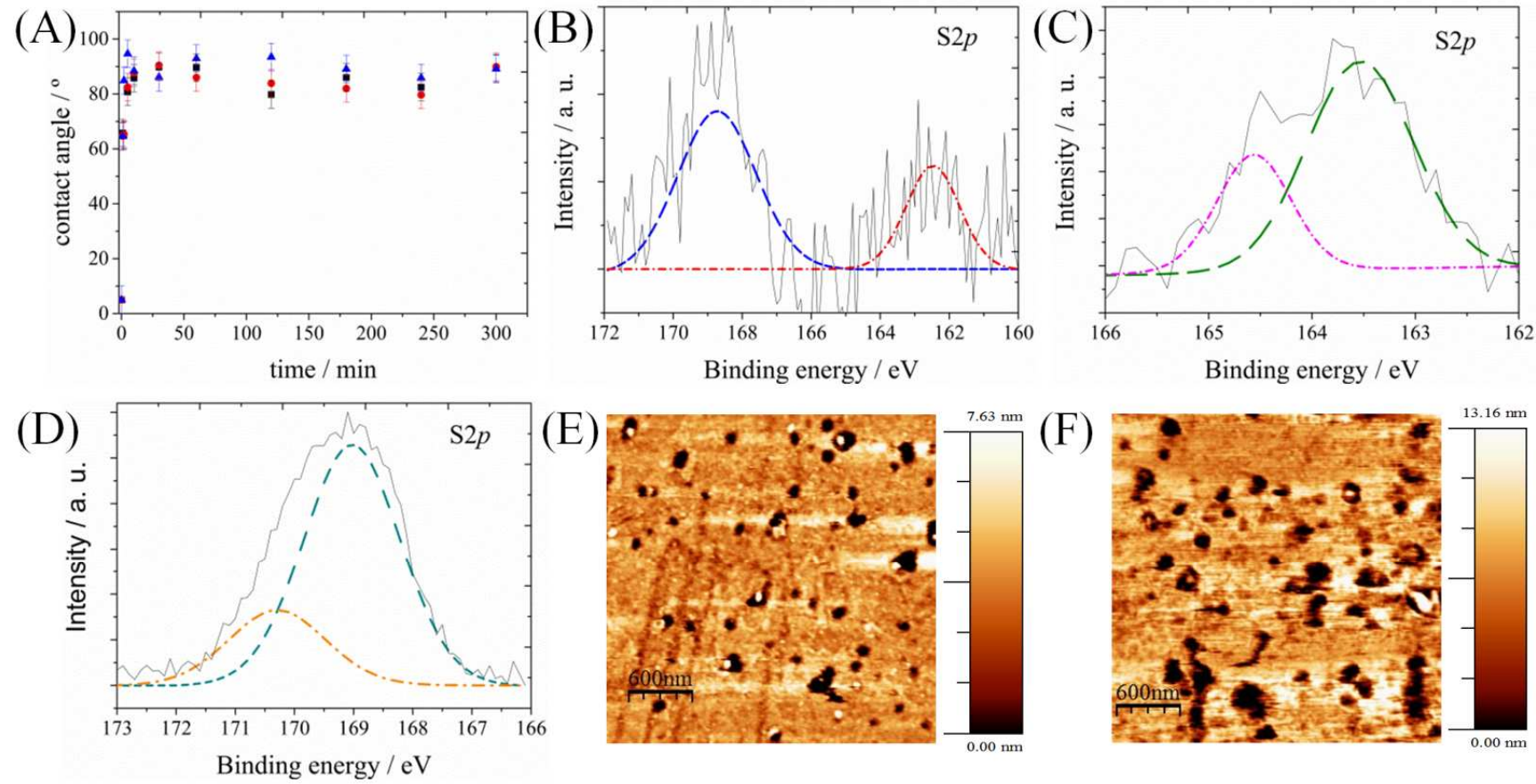

(G)
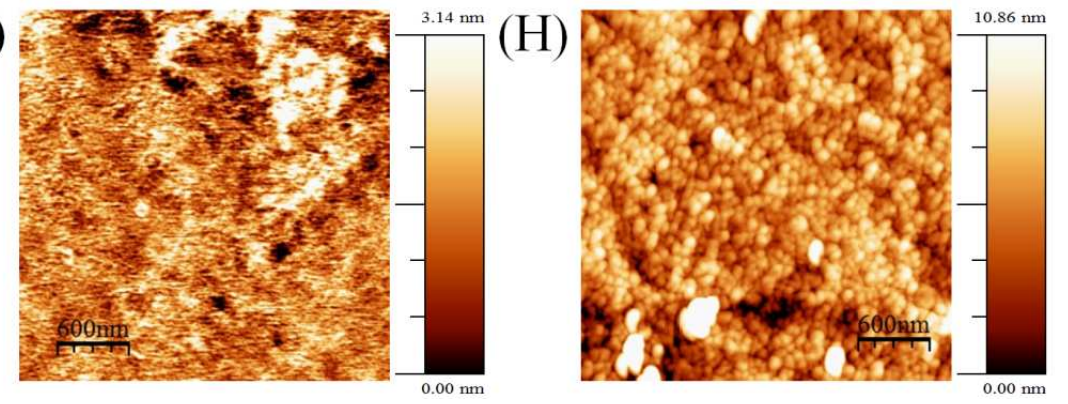

Fig. 1. (A) Variation of the ITO contact angle with immersion time in C12-SH $5 \mathrm{mM}$ (square), $50 \mathrm{mM}$ (circle) and pure (triangle). High-resolution S2 $p$ XPS spectra of C12-SH SAMs on ITO upon immersion in (B) $5 \mathrm{mM}$ C12-SH for $24 \mathrm{~h}$ and in (C,D) pure C12-SH for $5 \mathrm{~h}$. AFM topographical images of (E) bare ITO, (F) pretreated ITO and pretreated ITO samples after immersion in (G) $5 \mathrm{mM} \mathrm{C12-SH}$ for $24 \mathrm{~h}$ and in $(\mathrm{H})$ pure C12-SH for 5 h; Image size: $3 \mu \mathrm{m} \times 3 \mu \mathrm{m}$. 

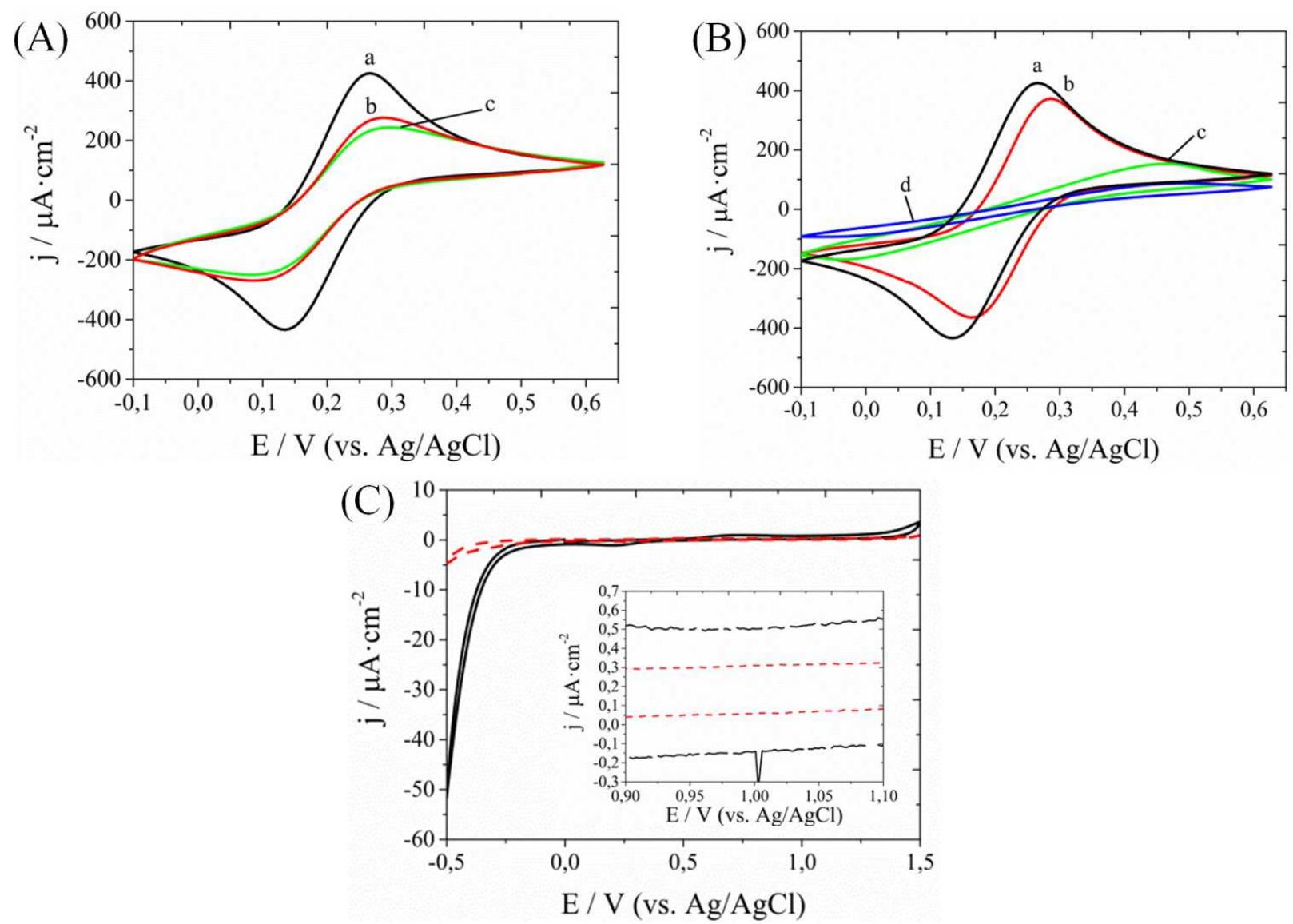

Fig. 2. Cyclic voltammograms of ITO samples in a $2 \mathrm{mM} \mathrm{Fe}(\mathrm{CN})_{6}^{3-/ 4-}$ solution with $0.2 \mathrm{M} \mathrm{KNO}_{3}$ after incubation in (A) $5 \mathrm{mM} \mathrm{C12-SH}$ for a) $0 \mathrm{~min}$, b) $2 \mathrm{~h}$ and c) $5 \mathrm{~h}$; and in (B) pure C12-SH for a) $0 \mathrm{~min}$, b) $2 \mathrm{~h}, \mathrm{c}) 5$ $\mathrm{h}$ and d) $6 \mathrm{~h}$. Scan rate $=50 \mathrm{mV} \cdot \mathrm{s}^{-1}$. (C) Cyclic voltammograms of bare ITO (solid line) and ITO-SAM (dashed line) in $\mathrm{H}_{2} \mathrm{SO}_{4} 0.05 \mathrm{M}$. Inset: zoom of (C) in the $0.9-1.1 \mathrm{~V}$ range. Scan rate $=50 \mathrm{mV} \cdot \mathrm{s}^{-1}$. 


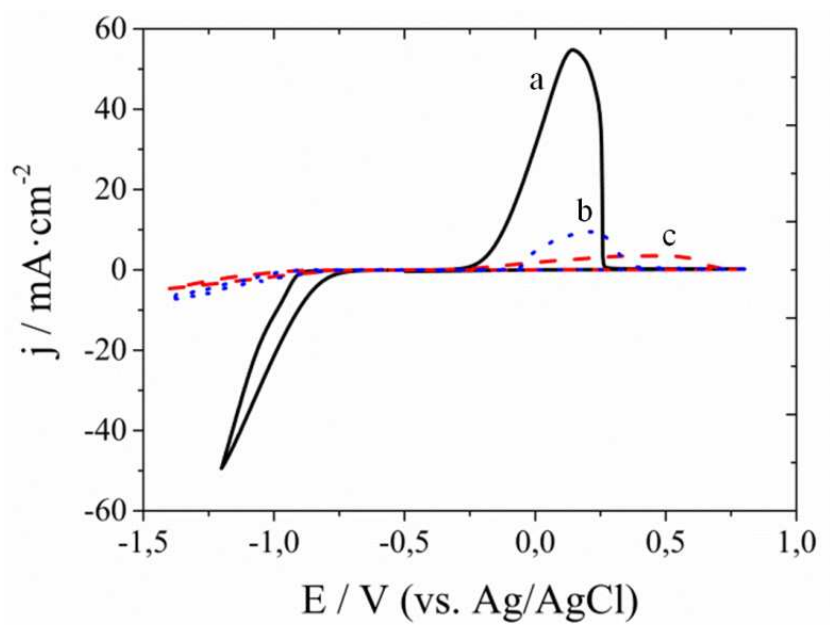

Fig. 3. Cyclic voltammogram, at $20 \mathrm{mV} \cdot \mathrm{s}^{-1}$, of a) bare ITO, b) ITO-SAM, and c) colloidal Ag-coated ITO-SAM in the CoNi electrolytic bath. 

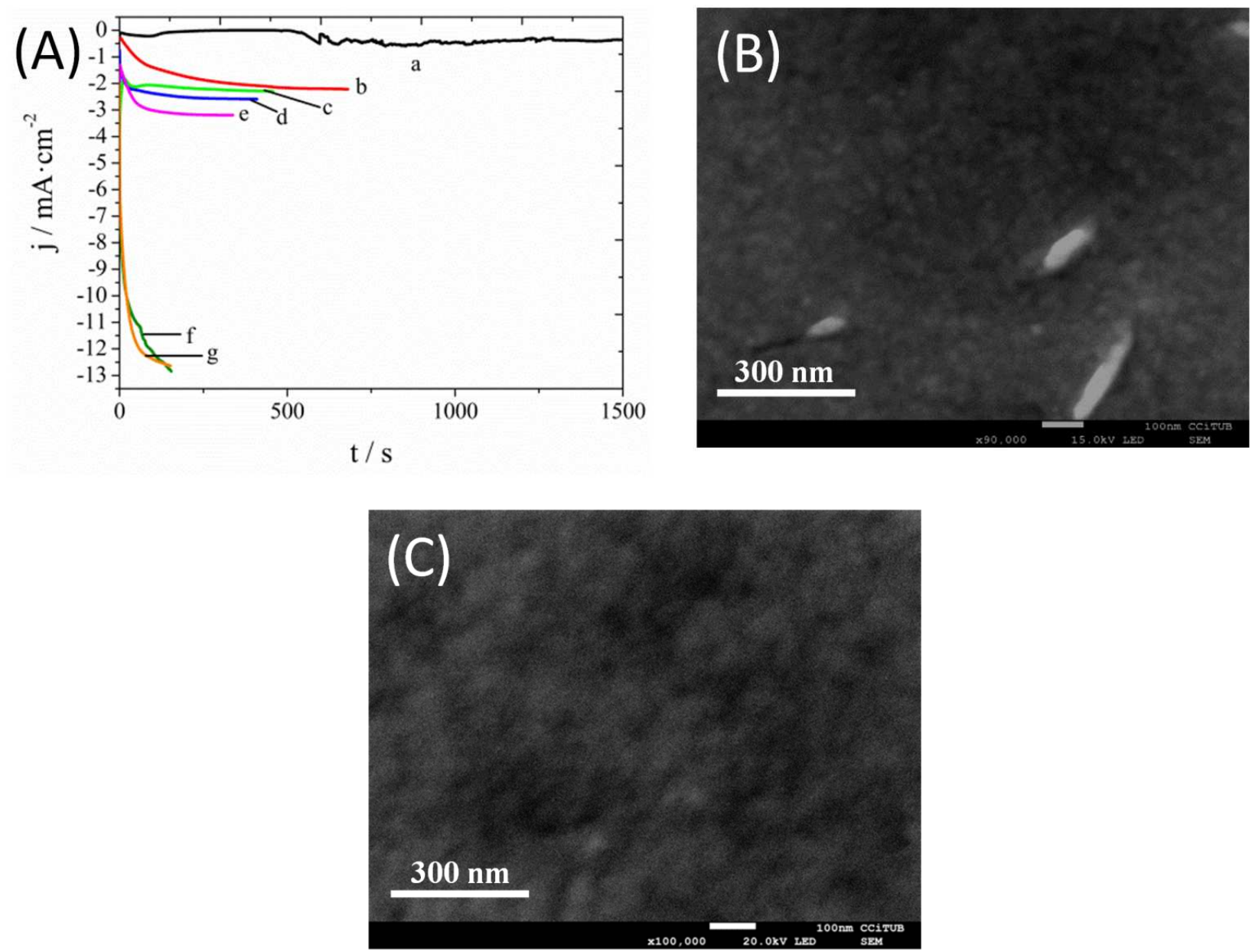

Fig. 4. (A) Chronoamperometric curves of CoNi deposition for a) ITO-SAM (E $\left.=-0.78 \mathrm{~V},<\sigma_{\mathrm{q}}>=-1 \mathrm{C} \cdot \mathrm{cm}^{-2}\right)$, b) ITO $\left.\left(E=-0.78 \mathrm{~V},<\sigma_{\mathrm{q}}>=-1.25 \mathrm{C} \cdot \mathrm{cm}^{-2}\right), \mathrm{c}\right)$ ITO-SAM-Pt $\left.\left(\mathrm{E}=-0.88 \mathrm{~V},<\sigma_{\mathrm{q}}>=-1 \mathrm{C} \cdot \mathrm{cm}^{-2}\right), \mathrm{d}\right)$ ITO-SAM $(\mathrm{E}=-0.92$ $\left.\mathrm{V},<\sigma_{\mathrm{q}}>=-1 \mathrm{C} \cdot \mathrm{cm}^{-2}\right)$, e) ITO-SAM-Pt $\left.\left(\mathrm{E}=-0.92 \mathrm{~V},<\sigma_{\mathrm{q}}>=-1 \mathrm{C} \cdot \mathrm{cm}^{-2}\right), \mathrm{f}\right)$ ITO-SAM $\left(\mathrm{E}=-1.1 \mathrm{~V},<\sigma_{\mathrm{q}}>=\right.$ $\left.1.75 \mathrm{C} \cdot \mathrm{cm}^{-2}\right)$ and $\left.\mathrm{g}\right)$ ITO $\left(\mathrm{E}=-0.94 \mathrm{~V},<\sigma_{\mathrm{q}}>=-1.75 \mathrm{C} \cdot \mathrm{cm}^{-2}\right)$. FE-SEM micrographs of CoNi deposit over (B) ITO $\left(E=-0.78 \mathrm{~V},<\sigma_{\mathrm{q}}>=-1.25 \mathrm{C} \cdot \mathrm{cm}^{-2}, \% \mathrm{Co}=74 \%, \mathrm{x} 90000\right),(\mathrm{C})$ ITO-SAM $\left(\mathrm{E}=-0.92 \mathrm{~V},<\sigma_{\mathrm{q}}>=-1 \mathrm{C} \cdot \mathrm{cm}^{-2}, \% \mathrm{Co}=\right.$ $74 \%, \mathrm{x} 100000)$ 

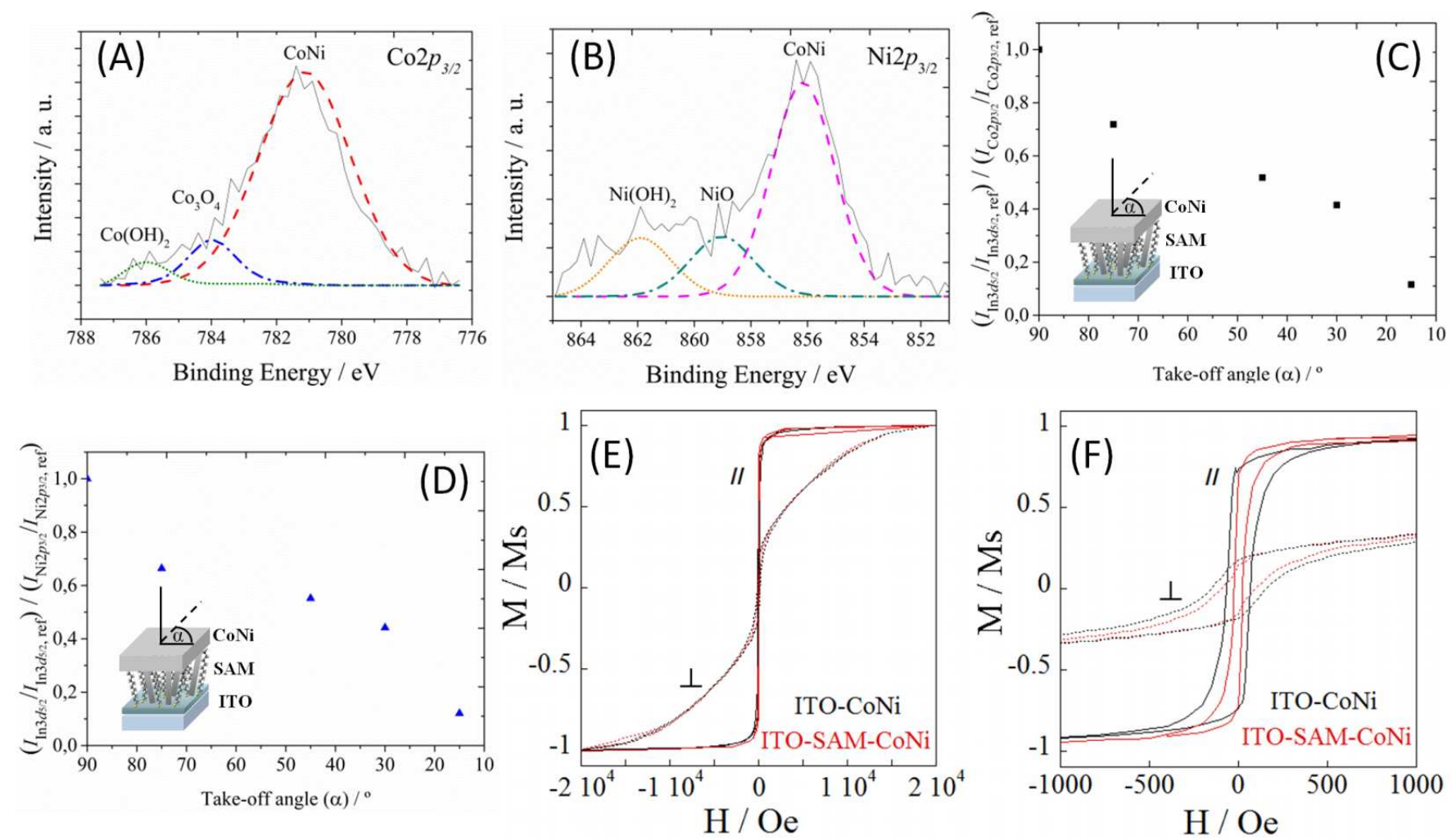

Fig. 5. High-resolution XPS spectra of a CoNi deposit over ITO-SAM in the (A) Co2 $2 p_{3 / 2}$ and (B) Ni2 $p_{3 / 2}$ region. Angular dependence of the normalized XPS intensity ratios of (C) In $3 d_{5 / 2}$ vs. Co2 $p_{3 / 2}$ and (D) In $3 d_{5 / 2} \mathrm{Vs}$. Ni2 $p_{3 / 2}$. $(\mathrm{E}, \mathrm{F})$ Hysteresis magnetic curves of the CoNi-ITO and CoNi-SAM-ITO structures, under application of a parallel (II) and perpendicular ( $\perp$ ) magnetic field. 

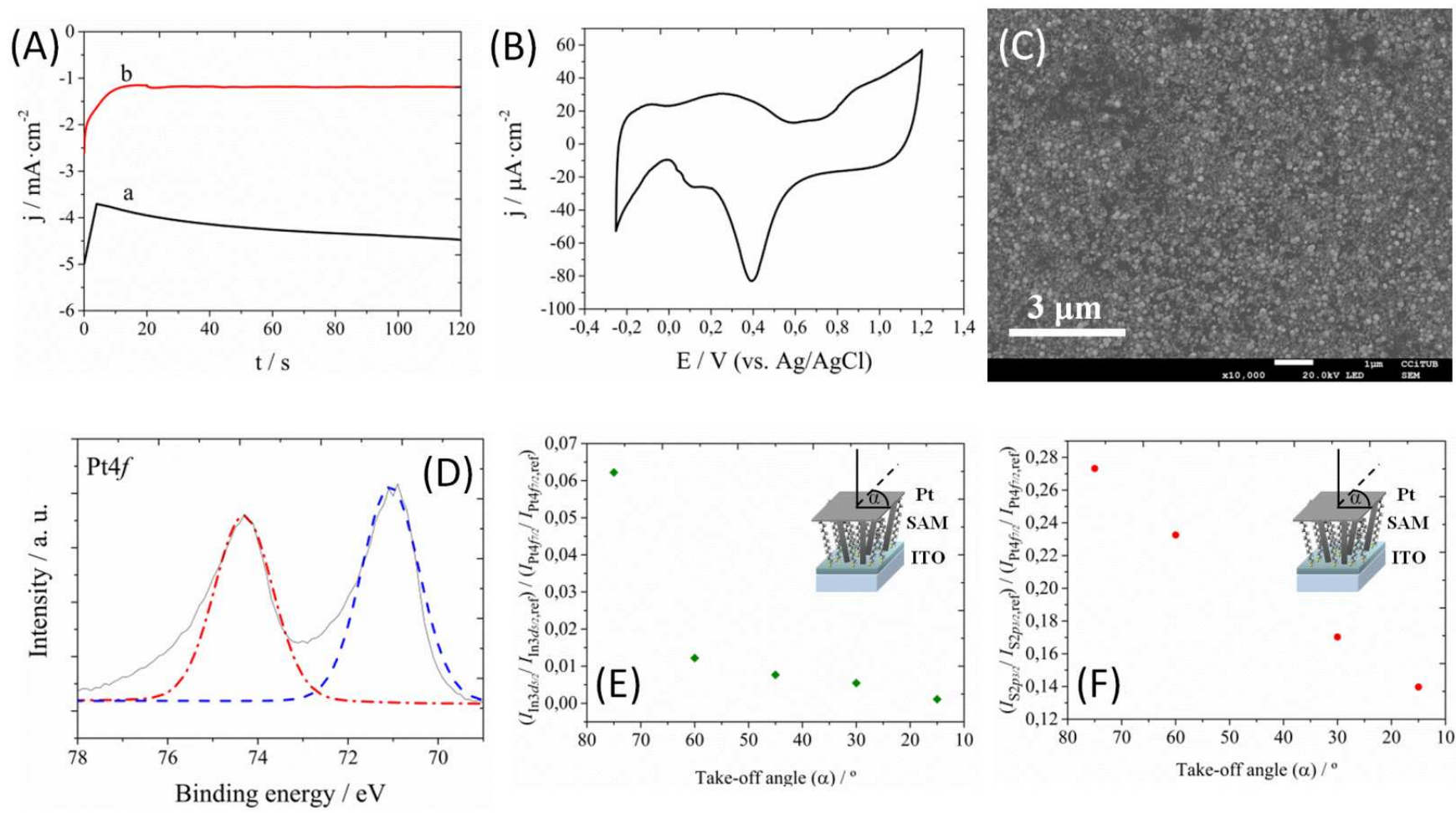

Fig. 6. (A) Chronoamperometric curves of $\mathrm{Pt}$ deposition for in $\mathrm{Na}_{2} \mathrm{PtCl}_{6} 5 \mathrm{mM}$ for a) bare ITO and b) ITO-SAM $(\mathrm{E}=-0.32 \mathrm{~V}, \mathrm{t}=120 \mathrm{~s})$. (B) Cyclic voltammogram in $\mathrm{H}_{2} \mathrm{SO}_{4} 0.05 \mathrm{M}$, at $20 \mathrm{mV} \cdot \mathrm{s}^{-1}$, of Pt nuclei directly electrodeposited over ITO-SAM $\left(<\sigma_{\mathrm{q}}>=-0.05 \mathrm{C} \cdot \mathrm{cm}^{-2}\right)$. (C) FE-SEM micrograph of Pt nuclei directly electrodeposited over ITO-SAM $\left(E=-0.32 \mathrm{~V},<\sigma_{\mathrm{q}}>=-0.05 \mathrm{C} \cdot \mathrm{cm}^{-2}, \mathrm{x} 10000\right)$. (D) High-resolution XPS spectrum of Pt nuclei over ITO-SAM in the Pt4 $f$ region. Angular dependence of the normalized XPS intensity ratios of (E) In $3 d_{5 / 2}$ vs. Pt $4 f_{7 / 2}$ and (F) S $2 p_{3 / 2}$ vs. Pt $4 f_{7 / 2}$. 


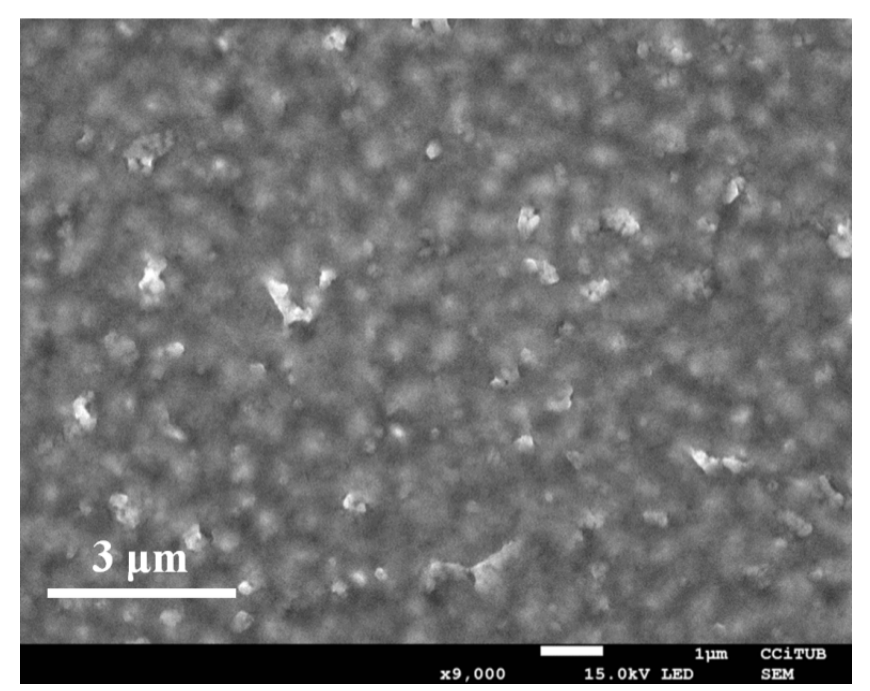

Fig. 7. FE-SEM micrograph of a CoNi deposit over ITO-SAM-Pt $\left(\mathrm{E}=-0.88 \mathrm{~V},<\sigma_{\mathrm{q}}>=-0.05 \mathrm{C} \cdot \mathrm{cm}^{-2}, \% \mathrm{Co}=66 \%\right.$, $\% \mathrm{Pt}=6 \%, \mathrm{x} 9000)$. 


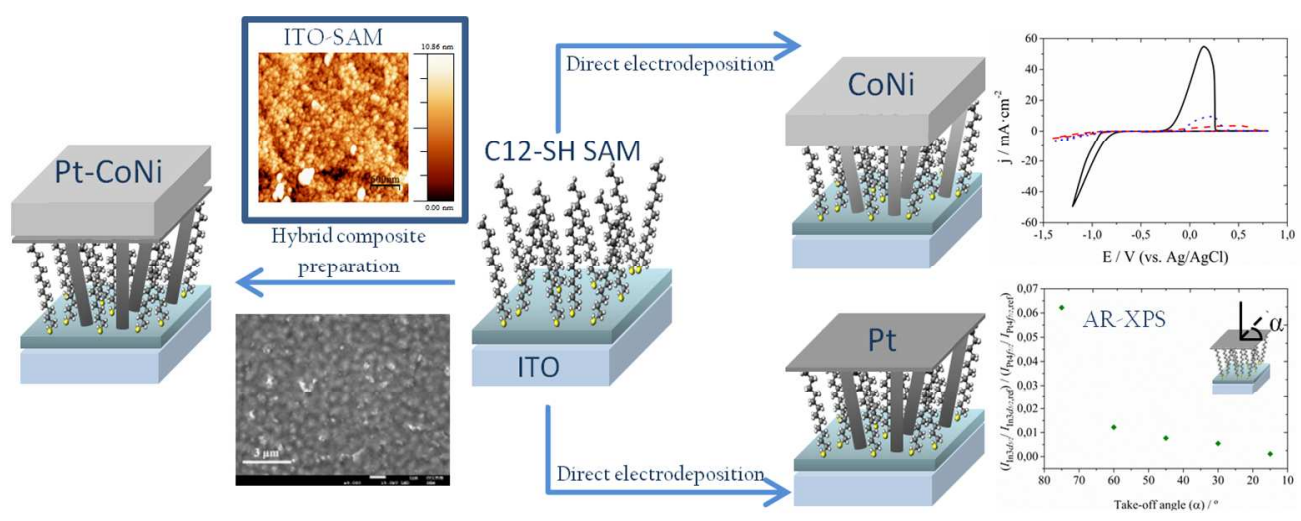

Graphical Abstract 129x49mm (300 x 300 DPI) 

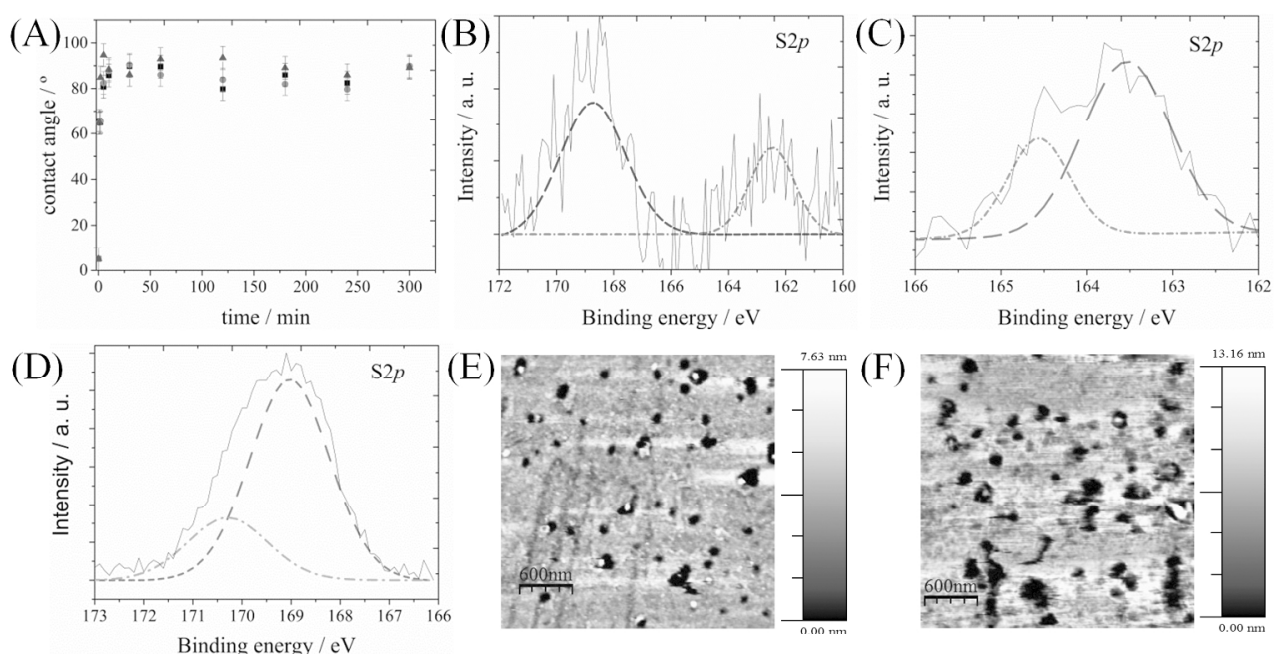

(E) $\bullet \div \%$ (F)

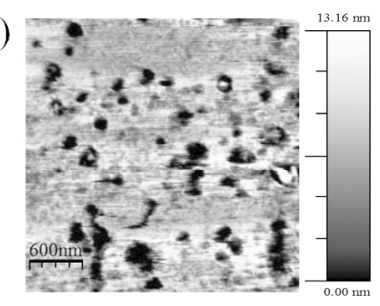

(G)
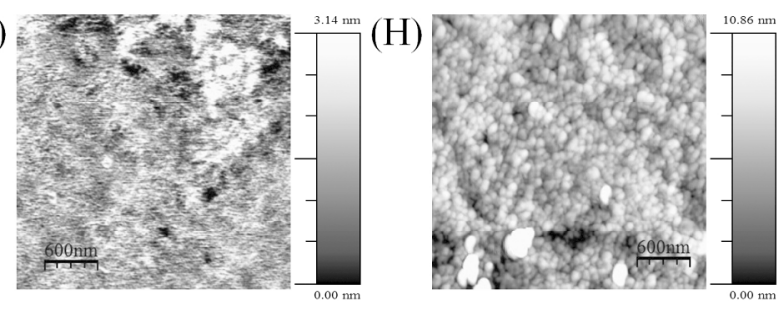

Figure 1 black and white $189 \times 150 \mathrm{~mm}$ (300 x 300 DPI) 

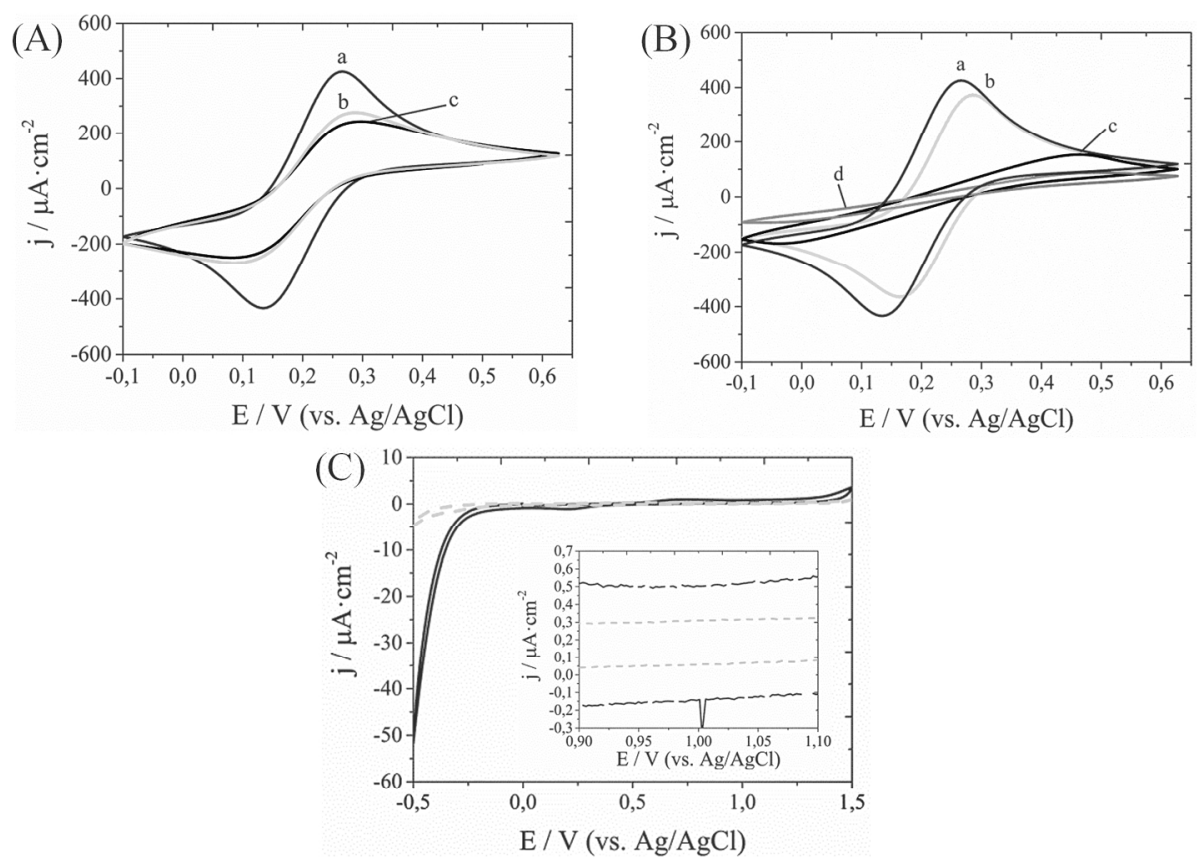

Figure 2 black and white $189 \times 190 \mathrm{~mm}$ ( $300 \times 300$ DPI) 


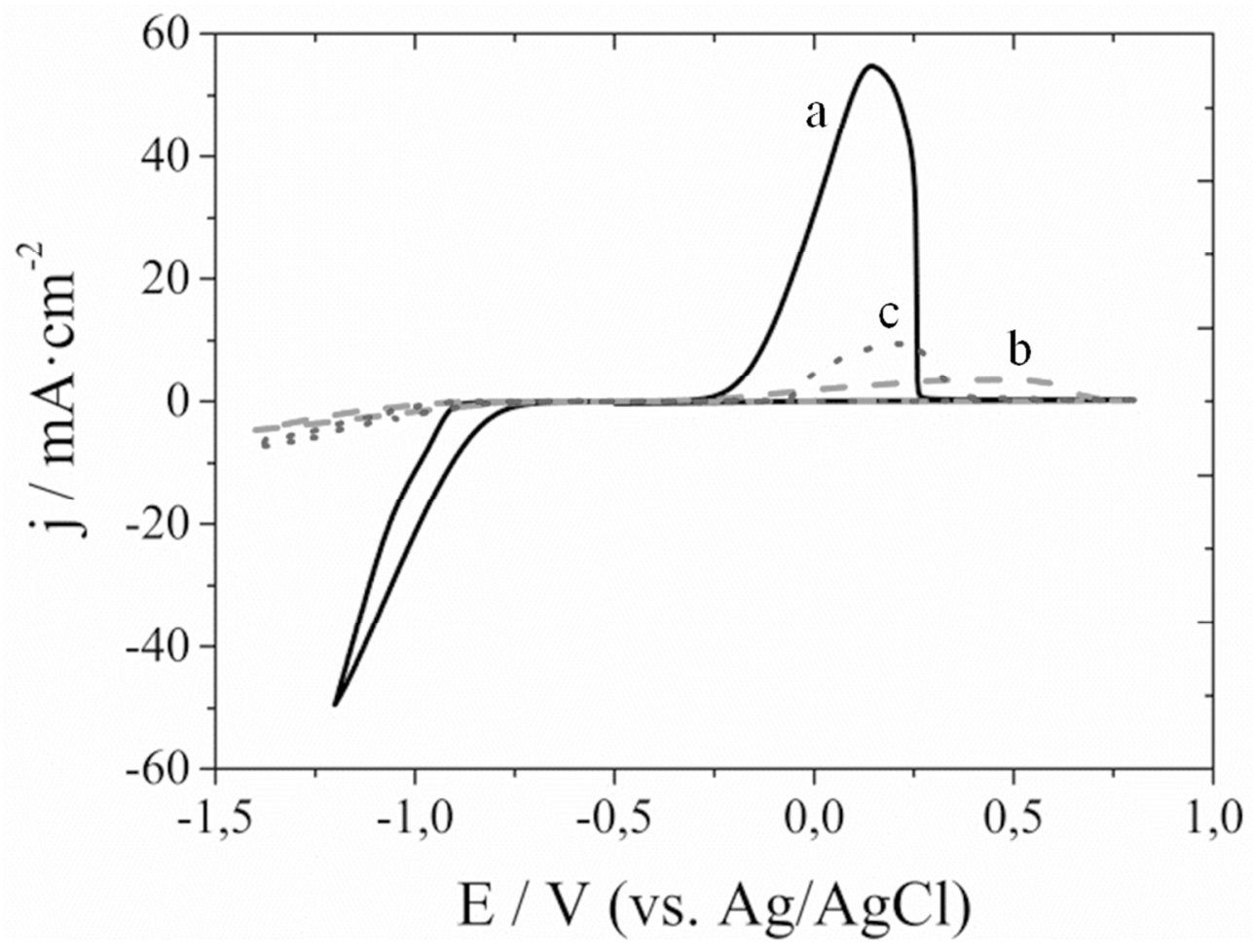

Figure 3 in black and white $90 \times 70 \mathrm{~mm}(300 \times 300$ DPI $)$ 

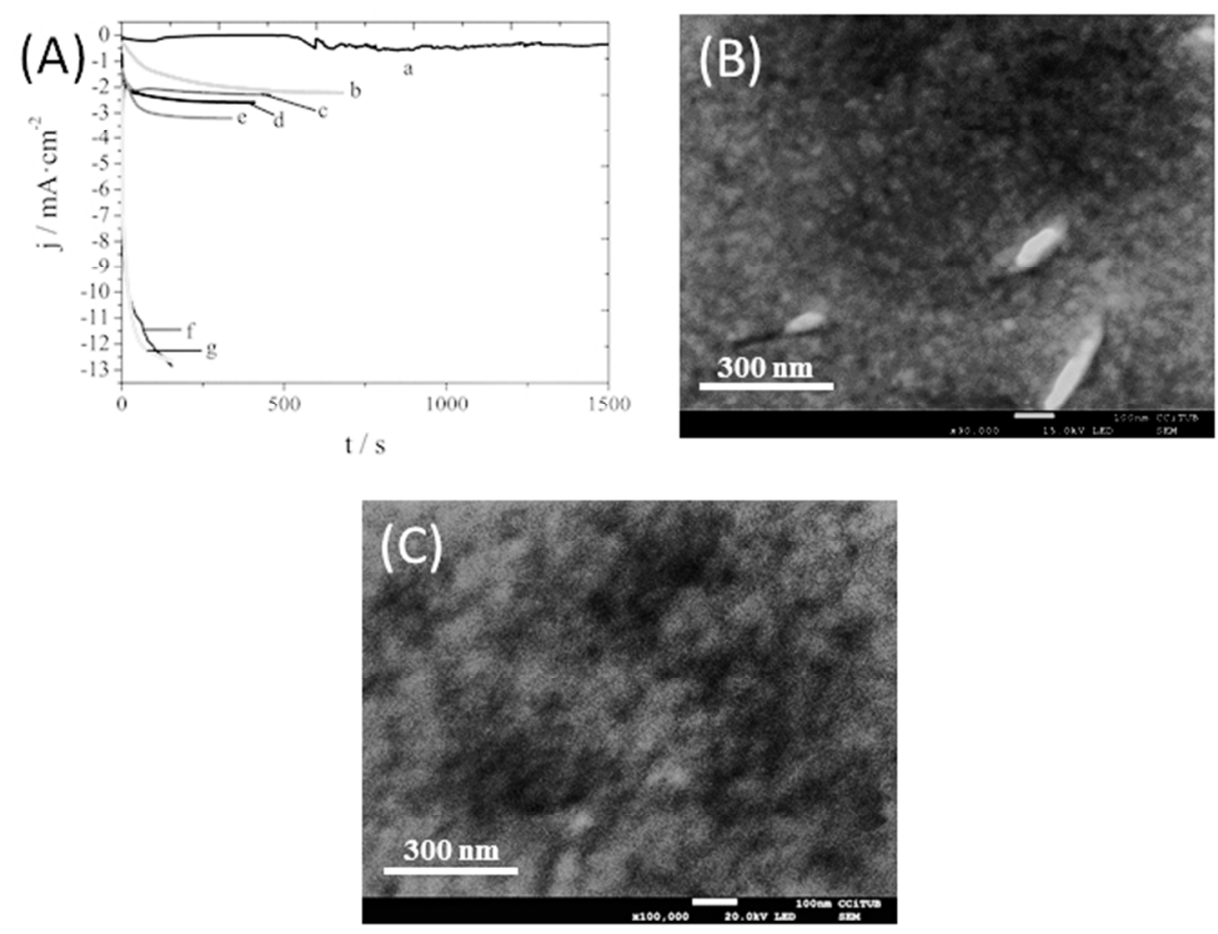

Figure 4 in black and white $189 \times 139 \mathrm{~mm}$ ( $96 \times 96 \mathrm{DPI})$ 

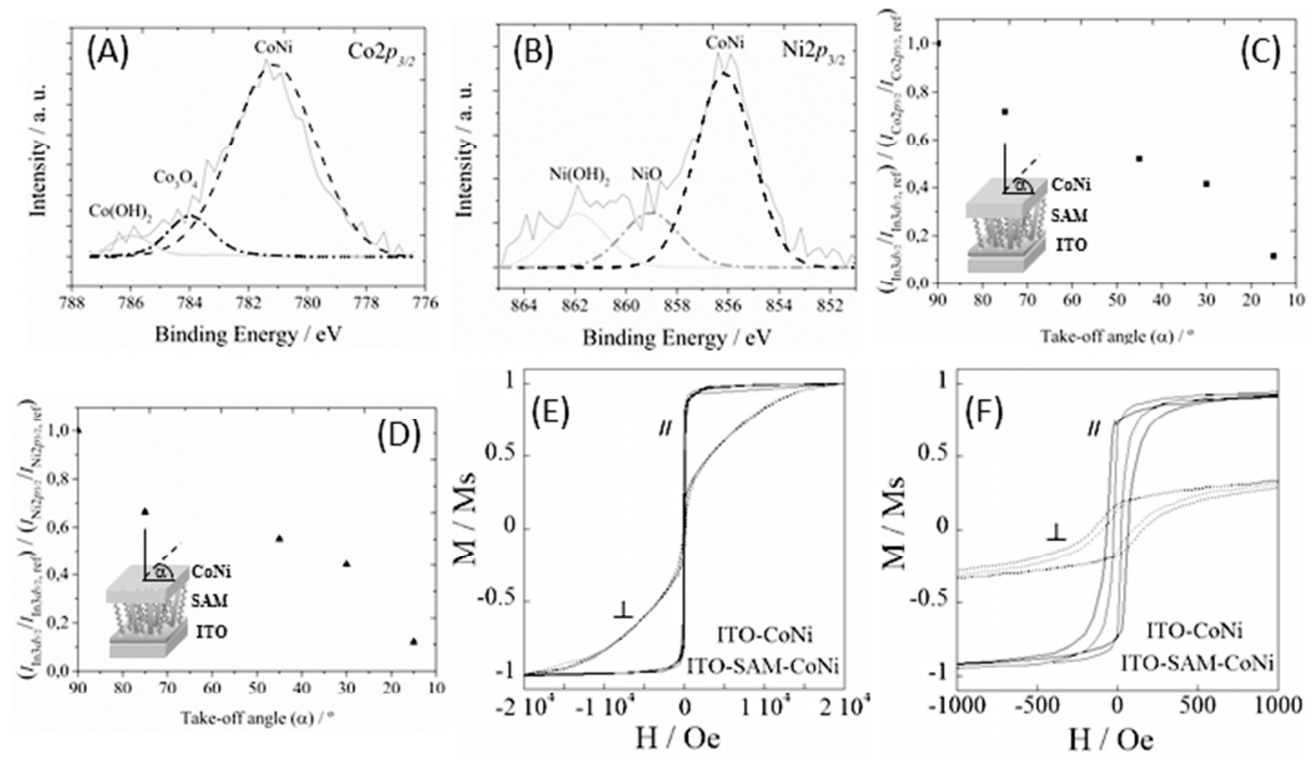

Figure 5 in black and white $189 \times 119 m m$ (96 x 96 DPI) 

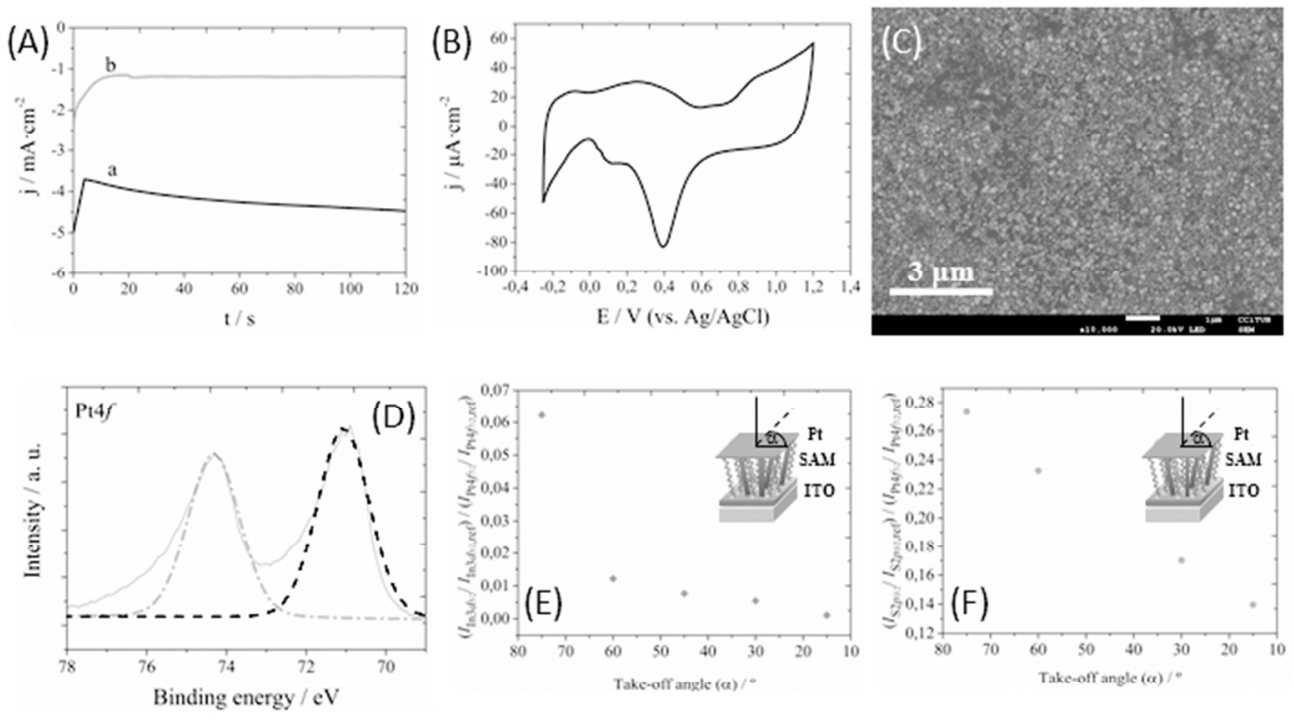

Figure 6 in black and white $189 \times 119 \mathrm{~mm}$ (96 x 96 DPI) 


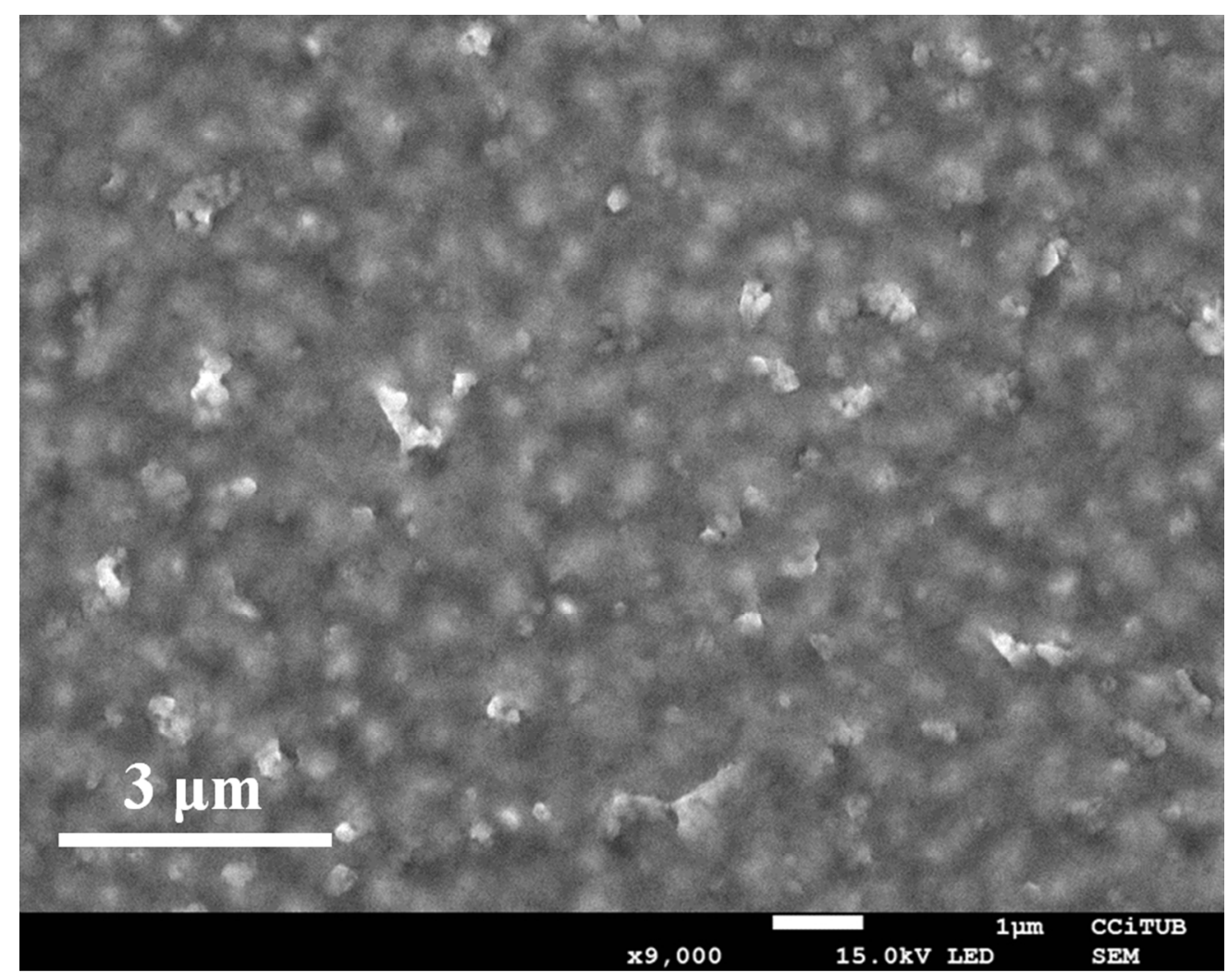

Figure 7

$90 \times 71 \mathrm{~mm}(300 \times 300$ DPI $)$ 